Structural Changes of Spinel $\mathrm{MCo}_{2} \mathrm{O}_{4}(\mathrm{M}=\mathrm{Mn}, \mathrm{Fe}$,

$\mathrm{Co}, \mathrm{Ni}$, and $\mathrm{Zn}$ ) Electrocatalysts during the Oxygen Evolution Reaction Investigated by In Situ X-ray Absorption Spectroscopy

\author{
Masafumi Harada,* Fukue Kotegawa, and Masako Kuwa
}

Department of Computer Science and Clothing Environment, Faculty of Human Life and Environment, Nara Women's University, Nara 630-8506, Japan

e-mail address: harada@cc.nara-wu.ac.jp (M. Harada). 


\section{Electrochemical characterizations}

The electrochemical active surface area (ECSA) is evaluated by the electrochemical double-layer capacitance $\left(\mathrm{C}_{\mathrm{dl}}\right)$ of catalysts on the working electrode. The $\mathrm{C}_{\mathrm{dl}}$ is determined from the non-Faradaic capacitive current, according to the following equation, associated with the scan-rate dependence of cyclic voltammograms (CVs). ${ }^{1-3}$

$\mathrm{C}_{\mathrm{dl}}=\Delta \mathrm{J} / 2 \mathrm{v}$

where $\Delta \mathrm{J}$ is the current density difference value of charging and discharging current densities $\left(\mathrm{mA} / \mathrm{cm}^{2}\right)$ and $v$ is the scan rate $(\mathrm{mV} / \mathrm{s})$. The potential range of the measurements is from 0.95 to $1.0 \mathrm{~V}$ (vs. RHE). By plotting the current density at the middle potential of $0.975 \mathrm{~V}$ (vs. RHE) against the scan rate, a linear curve is obtained. The linear slope is equal to the twice of $\mathrm{C}_{\mathrm{dl}}$, which is used to estimate the roughness factor $\left(\mathrm{R}_{\mathrm{f}}\right)$. The scan rates are from 2 to $100 \mathrm{mV} / \mathrm{s}(2,5,10,25,50$, and $100 \mathrm{mV} / \mathrm{s}$ ).

$\mathrm{R}_{\mathrm{f}}=\mathrm{C}_{\mathrm{dl}} / \mathrm{C}_{\mathrm{s}}$

where $\mathrm{C}_{\mathrm{s}}$ is the specific capacitance value of a smooth surface for transition-metal oxide in alkaline solution. The standard value of $\mathrm{C}_{\mathrm{s}}$ is used as $40 \mu \mathrm{F} / \mathrm{cm}^{2}$ to calculate the $\mathrm{R}_{\mathrm{f}}$ values. ${ }^{1}$ 
Table S1. Spectral Fitting Parameters for Co 2p XPS Spectra of Various Cation-substituted $\mathrm{Co}_{3} \mathrm{O}_{4}$

\begin{tabular}{|c|c|c|c|c|c|c|c|c|c|c|}
\hline \multirow[b]{2}{*}{ sample } & \multicolumn{4}{|c|}{$2 \mathrm{p}_{3 / 2}$} & \multicolumn{4}{|c|}{$2 \mathrm{p}_{1 / 2}$} & \multicolumn{2}{|c|}{ contribution / \% } \\
\hline & $\mathrm{Co}^{2+}$ & $\mathrm{Co}^{3+}$ & $\begin{array}{c}\mathrm{Co}^{2+} \\
\text { satellite } \\
\end{array}$ & $\begin{array}{c}\mathrm{Co}^{3+} \\
\text { satellite } \\
\end{array}$ & $\mathrm{Co}^{2+}$ & $\mathrm{Co}^{3+}$ & $\begin{array}{c}\mathrm{Co}^{2+} \\
\text { satellite } \\
\end{array}$ & $\begin{array}{c}\mathrm{Co}^{3+} \\
\text { satellite } \\
\end{array}$ & $\mathrm{Co}^{2+}$ & $\mathrm{Co}^{3+}$ \\
\hline $\mathrm{Co}_{3} \mathrm{O}_{4}$ & $\begin{array}{l}781.4 \\
(2.6)\end{array}$ & $\begin{array}{l}779.5 \\
(2.0)\end{array}$ & $\begin{array}{c}785.2 \\
(3.5)\end{array}$ & $\begin{array}{c}788.8 \\
(3.9)\end{array}$ & $\begin{array}{c}796.8 \\
(3.3)\end{array}$ & $\begin{array}{l}794.6 \\
(2.4)\end{array}$ & $\begin{array}{c}801.8 \\
(3.5)\end{array}$ & $\begin{array}{c}804.8 \\
(3.7)\end{array}$ & 36 & 64 \\
\hline $\mathrm{MnCo}_{2} \mathrm{O}_{4}$ & $\begin{array}{l}781.7 \\
(2.4)\end{array}$ & $\begin{array}{l}780.0 \\
(1.6)\end{array}$ & $\begin{array}{l}784.8 \\
(3.8)\end{array}$ & $\begin{array}{l}788.7 \\
(4.9)\end{array}$ & $\begin{array}{c}797.1 \\
(2.7)\end{array}$ & $\begin{array}{l}795.2 \\
(1.9)\end{array}$ & $\begin{array}{c}801.6 \\
(4.0)\end{array}$ & $\begin{array}{c}804.7 \\
(4.1)\end{array}$ & 40 & 60 \\
\hline $\mathrm{FeCo}_{2} \mathrm{O}_{4}$ & $\begin{array}{l}781.9 \\
(2.8)\end{array}$ & $\begin{array}{l}780.0 \\
(2.3)\end{array}$ & $\begin{array}{l}785.2 \\
(4.5)\end{array}$ & $\begin{array}{l}789.5 \\
(4.4)\end{array}$ & $\begin{array}{c}797.2 \\
(3.0)\end{array}$ & $\begin{array}{l}795.1 \\
(2.5)\end{array}$ & $\begin{array}{c}801.5 \\
(4.2)\end{array}$ & $\begin{array}{c}805.5 \\
(4.5)\end{array}$ & 40 & 60 \\
\hline $\mathrm{NiCo}_{2} \mathrm{O}_{4}$ & $\begin{array}{l}781.3 \\
(2.4)\end{array}$ & $\begin{array}{l}779.4 \\
(2.0)\end{array}$ & $\begin{array}{c}784.8 \\
(4.0)\end{array}$ & $\begin{array}{l}788.7 \\
(3.3)\end{array}$ & $\begin{array}{c}796.9 \\
(3.2)\end{array}$ & $\begin{array}{l}794.6 \\
(2.4)\end{array}$ & $\begin{array}{c}801.7 \\
(4.5)\end{array}$ & $\begin{array}{c}804.7 \\
(3.4)\end{array}$ & 35 & 65 \\
\hline $\mathrm{ZnCo}_{2} \mathrm{O}_{4}$ & $\begin{array}{l}781.2 \\
(2.4)\end{array}$ & $\begin{array}{c}779.3 \\
(2.1)\end{array}$ & $\begin{array}{c}785.2 \\
(5.0)\end{array}$ & $\begin{array}{c}788.7 \\
(3.3)\end{array}$ & $\begin{array}{c}796.8 \\
(2.7)\end{array}$ & $\begin{array}{c}794.5 \\
(2.4)\end{array}$ & $\begin{array}{c}802.0 \\
(4.0)\end{array}$ & $\begin{array}{c}804.7 \\
(3.0)\end{array}$ & 24 & 76 \\
\hline
\end{tabular}

The numbers in parenthesis denote the full width at half-maximum $(\mathrm{eV})$. 
Table S2. Spectral Fitting Parameters for M 2p (M = Mn, Co, Ni, and Zn) XPS Spectra of Various Cation-substituted Co $\mathrm{O}_{4}$

\begin{tabular}{|c|c|c|c|c|c|c|c|c|c|c|}
\hline \multirow[b]{2}{*}{ sample } & \multicolumn{4}{|c|}{$2 \mathrm{p}_{3 / 2}$} & \multicolumn{4}{|c|}{$2 \mathrm{p}_{1 / 2}$} & \multicolumn{2}{|c|}{ contribution / \% } \\
\hline & $\mathrm{M}^{2+}$ & $\mathrm{M}^{3+}$ & surface & $\begin{array}{c}\mathrm{M}^{2+}, \mathrm{M}^{3+} \\
\text { satellite }\end{array}$ & $\mathrm{M}^{2+}$ & $\mathrm{M}^{3+}$ & surface & $\begin{array}{c}\mathrm{M}^{2+}, \mathrm{M}^{3+} \\
\text { satellite }\end{array}$ & $\mathrm{M}^{2+}$ & $\mathrm{M}^{3+}$ \\
\hline $\mathrm{MnCo}_{2} \mathrm{O}_{4}$ & $\begin{array}{c}641.6 \\
(2.4)\end{array}$ & $\begin{array}{c}643.4 \\
(4.2)\end{array}$ & - & - & $\begin{array}{c}653.1 \\
(2.9)\end{array}$ & $\begin{array}{c}654.2 \\
(4.0)\end{array}$ & - & - & 55 & 45 \\
\hline $\mathrm{FeCo}_{2} \mathrm{O}_{4}$ & $\begin{array}{l}709.8 \\
(2.2)\end{array}$ & $\begin{array}{l}711.3 \\
(2.4)\end{array}$ & $\begin{array}{l}713.3 \\
(3.6)\end{array}$ & $\begin{array}{c}718.1 \\
(6.5)\end{array}$ & $\begin{array}{l}722.9 \\
(2.9)\end{array}$ & $\begin{array}{c}724.5 \\
(3.0)\end{array}$ & $\begin{array}{l}726.5 \\
(4.3)\end{array}$ & $\begin{array}{c}731.8 \\
(6.5)\end{array}$ & 48 & 52 \\
\hline $\mathrm{NiCo}_{2} \mathrm{O}_{4}$ & $\begin{array}{c}854.1 \\
(2.1)\end{array}$ & $\begin{array}{c}855.8 \\
(2.2)\end{array}$ & - & $\begin{array}{c}861.1 \\
(7.0)\end{array}$ & $\begin{array}{c}871.6 \\
(2.4)\end{array}$ & $\begin{array}{c}873.5 \\
(2.9)\end{array}$ & - & $\begin{array}{c}879.3 \\
(6.0)\end{array}$ & 54 & 46 \\
\hline $\mathrm{ZnCo}_{2} \mathrm{O}_{4}$ & $\begin{array}{c}1020.8 \\
(2.1)\end{array}$ & - & - & - & $\begin{array}{c}1043.8 \\
(2.3)\end{array}$ & - & - & - & 100 & 0 \\
\hline
\end{tabular}

The numbers in parenthesis denote the full width at half-maximum $(\mathrm{eV})$. 
Table S3. Simulated Parameters of the Elements in Equivalent Circuits at the Potential of 1.0 V (vs. RHE) for Various Cation-substituted $\mathrm{Co}_{3} \mathrm{O}_{4}$

\begin{tabular}{|c|c|c|c|c|c|c|c|}
\hline \multirow{2}{*}{ sample } & \multirow[b]{2}{*}{$R_{\mathrm{S}}\left(\Omega \mathrm{cm}^{2}\right)$} & \multirow[b]{2}{*}{$R_{\mathrm{p}}\left(\Omega \mathrm{cm}^{2}\right)$} & \multicolumn{2}{|c|}{$Q_{\mathrm{dl}}$} & \multirow[b]{2}{*}{$R_{\mathrm{ad}}\left(\Omega \mathrm{cm}^{2}\right)$} & \multicolumn{2}{|l|}{$Q_{\mathrm{ad}}$} \\
\hline & & & $\begin{array}{c}Q_{\mathrm{dl}} \\
\left(\mu \mathrm{F} \mathrm{s} \mathrm{s}^{\mathrm{n}} \mathrm{cm}^{-2}\right)\end{array}$ & $n$ & & $\begin{array}{c}Q_{\mathrm{ad}} \\
\left(\mu \mathrm{F} \mathrm{s} \mathrm{s}^{\mathrm{n}} \mathrm{cm}^{-2}\right)\end{array}$ & $n$ \\
\hline $\mathrm{Co}_{3} \mathrm{O}_{4}$ & 1.21 & 4.71 & 1.77 & 0.980 & 39255 & 249.60 & 0.854 \\
\hline $\mathrm{MnCo}_{2} \mathrm{O}_{4}$ & 0.80 & 3.84 & 1.84 & 0.985 & 47440 & 737.24 & 0.857 \\
\hline $\mathrm{FeCo}_{2} \mathrm{O}_{4}$ & 1.11 & 5.25 & 1.89 & 0.975 & 39233 & 174.84 & 0.859 \\
\hline $\mathrm{NiCo}_{2} \mathrm{O}_{4}$ & 1.02 & 5.20 & 1.36 & 0.984 & 37948 & 458.48 & 0.816 \\
\hline $\mathrm{ZnCo}_{2} \mathrm{O}_{4}$ & 1.23 & 4.62 & 1.46 & 0.998 & 32040 & 500.65 & 0.839 \\
\hline
\end{tabular}


Table S4. Simulated Parameters of the Elements in Equivalent Circuits at the Potential of 1.6 V (vs. RHE) for Various Cation-substituted $\mathrm{Co}_{3} \mathrm{O}_{4}$

\begin{tabular}{|c|c|c|c|c|c|c|c|}
\hline \multirow{2}{*}{ sample } & \multirow[b]{2}{*}{$R_{\mathrm{S}}\left(\Omega \mathrm{cm}^{2}\right)$} & \multirow[b]{2}{*}{$R_{\mathrm{p}}\left(\Omega \mathrm{cm}^{2}\right)$} & \multicolumn{2}{|c|}{$Q_{\mathrm{dl}}$} & \multirow[b]{2}{*}{$R_{\mathrm{ad}}\left(\Omega \mathrm{cm}^{2}\right)$} & \multicolumn{2}{|l|}{$Q_{\mathrm{ad}}$} \\
\hline & & & $\begin{array}{c}Q_{\mathrm{dl}} \\
\left(\mu \mathrm{F} \mathrm{s} \mathrm{s}^{\mathrm{n}} \mathrm{cm}^{-2}\right)\end{array}$ & $n$ & & $\begin{array}{c}Q_{\mathrm{ad}} \\
\left(\mu \mathrm{F} \mathrm{s} \mathrm{s}^{\mathrm{n}} \mathrm{cm}^{-2}\right)\end{array}$ & $n$ \\
\hline $\mathrm{Co}_{3} \mathrm{O}_{4}$ & 1.16 & 5.18 & 1.55 & 0.994 & 181130 & 4613.9 & 0.747 \\
\hline $\mathrm{MnCo}_{2} \mathrm{O}_{4}$ & 1.09 & 4.66 & 1.69 & 0.982 & 16593 & 544.95 & 0.756 \\
\hline $\mathrm{FeCo}_{2} \mathrm{O}_{4}$ & 1.15 & 5.34 & 1.78 & 0.983 & 26297 & 1293.1 & 0.623 \\
\hline $\mathrm{NiCo}_{2} \mathrm{O}_{4}$ & 1.09 & 4.88 & 2.12 & 0.968 & 209.13 & 2122.4 & 0.691 \\
\hline $\mathrm{ZnCo}_{2} \mathrm{O}_{4}$ & 1.08 & 4.42 & 1.60 & 0.991 & 165.74 & 351.28 & 0.875 \\
\hline
\end{tabular}


Table S5. Structural Parameters of $\mathrm{Co}_{3} \mathrm{O}_{4}$ Electrocatalysts Obtained from Co K-edge EXAFS Analysis

\begin{tabular}{|lcccc|ccccc|}
\hline \multicolumn{5}{c|}{ A site } & \multicolumn{5}{c|}{ B site } \\
\hline Bond & $r / \AA$ & $\mathrm{CN}$ & $\sigma^{2} / \AA^{2}$ & $\Delta E / \mathrm{eV}$ & Bond & $r / \AA$ & $\mathrm{CN}$ & $\sigma^{2} / \AA^{2}$ & $\Delta E / \mathrm{eV}$ \\
\hline $\mathrm{Co}_{\mathrm{A}}-\mathrm{O}_{\mathrm{A}}$ & 1.87 & 4.0 & 0.009 & 1.05 & $\mathrm{Co}_{\mathrm{B}}-\mathrm{O}_{\mathrm{B}}$ & 1.92 & 6.0 & 0.003 & -1.66 \\
$\mathrm{Co}_{\mathrm{A}}-\mathrm{Co}_{\mathrm{B}}$ & 3.37 & 12.0 & 0.006 & 4.50 & $\mathrm{Co}_{\mathrm{B}}-\mathrm{Co}_{\mathrm{B}}$ & 2.87 & 6.0 & 0.003 & 4.50 \\
$\mathrm{Co}_{\mathrm{A}}-\mathrm{O}_{\mathrm{B}}$ & 3.45 & 12.0 & 0.002 & 1.05 & $\mathrm{Co}_{\mathrm{B}}-\mathrm{Co}_{\mathrm{A}}$ & 3.37 & 6.0 & 0.006 & 4.50 \\
$\mathrm{Co}_{\mathrm{A}}-\mathrm{Co}_{\mathrm{A}}$ & 3.55 & 4.0 & 0.003 & 4.50 & & & & & \\
\hline
\end{tabular}

${ }^{a}$ Coordination numbers $(\mathrm{CN})$ were fixed.

${ }^{b}$ The value of the $R$-factor is $2.4 \%$ at the Co K-edge.

${ }^{c}$ The degree of inversion cannot be quantitatively determined by the fitting of EXAFS data, because EXAFS oscillations of $\mathrm{Co}^{2+}$ and $\mathrm{Co}^{3+}$ are very similar so that they are not distinguishable each other.

Table S6. Structural Parameters of $\mathrm{MnCo}_{2} \mathrm{O}_{4}$ Electrocatalysts Obtained from Co and Mn K-edge EXAFS Analysis

\begin{tabular}{|c|c|c|c|c|c|c|c|c|c|}
\hline \multicolumn{10}{|l|}{ Co K-edge } \\
\hline \multicolumn{5}{|c|}{ A site $\left(x_{\mathrm{A}}=0.57\right)$} & \multicolumn{5}{|c|}{$\mathrm{B}$ site $\left(x_{\mathrm{B}}=0.43\right)$} \\
\hline Bond & $r / \AA$ & $\mathrm{CN}$ & $\sigma^{2} / \AA^{2}$ & $\Delta E / \mathrm{eV}$ & Bond & $r / \AA$ & $\mathrm{CN}$ & $\sigma^{2} / \AA^{2}$ & $\Delta E / \mathrm{eV}$ \\
\hline $\mathrm{Co}_{\mathrm{A}}-\mathrm{O}_{\mathrm{A}}$ & 1.85 & 4.0 & 0.013 & 9.95 & $\mathrm{Co}_{\mathrm{B}}-\mathrm{O}_{\mathrm{B}}$ & 1.92 & 6.0 & 0.004 & -1.21 \\
\hline $\mathrm{Co}_{\mathrm{A}^{-}}-\mathrm{Co}_{\mathrm{B}}$ & 3.38 & 12.0 & 0.009 & 0.65 & $\mathrm{Co}_{\mathrm{B}}-\mathrm{Co}_{\mathrm{B}}$ & 2.86 & 6.0 & 0.007 & 0.65 \\
\hline $\mathrm{Co}_{\mathrm{A}}-\mathrm{O}_{\mathrm{B}}$ & 3.39 & 12.0 & 0.007 & -10.00 & $\mathrm{Co}_{\mathrm{B}}-\mathrm{Co}_{\mathrm{A}}$ & 3.38 & 6.0 & 0.009 & 0.65 \\
\hline $\mathrm{Co}_{\mathrm{A}^{-}} \mathrm{Co}_{\mathrm{A}}$ & 3.65 & 4.0 & 0.008 & 0.65 & & & & & \\
\hline \multicolumn{10}{|l|}{ Mn K-edge } \\
\hline \multicolumn{5}{|c|}{ A site $\left(x_{\mathrm{A}}=0.63\right)$} & \multicolumn{5}{|c|}{$\mathrm{B}$ site $\left(x_{\mathrm{B}}=0.37\right)$} \\
\hline Bond & $r / \AA$ & $\mathrm{CN}$ & $\sigma^{2} / \AA^{2}$ & $\Delta E / \mathrm{eV}$ & Bond & $r / \AA$ & $\mathrm{CN}$ & $\sigma^{2} / \AA^{2}$ & $\Delta E / \mathrm{eV}$ \\
\hline $\mathrm{Mn}_{\mathrm{A}}-\mathrm{O}_{\mathrm{A}}$ & 1.89 & 4.0 & 0.003 & 4.38 & $\mathrm{Mn}_{\mathrm{B}}-\mathrm{O}_{\mathrm{B}}$ & 2.07 & 6.0 & 0.020 & -5.64 \\
\hline $\mathrm{Mn}_{\mathrm{A}}-\mathrm{Mn}_{\mathrm{B}}$ & 3.34 & 12.0 & 0.016 & 7.33 & $\mathrm{Mn}_{\mathrm{B}}-\mathrm{Mn}_{\mathrm{B}}$ & 2.82 & 6.0 & 0.008 & -7.41 \\
\hline $\mathrm{Mn}_{\mathrm{A}}-\mathrm{O}_{\mathrm{B}}$ & 3.43 & 12.0 & 0.004 & 3.21 & $\mathrm{Mn}_{\mathrm{B}}-\mathrm{Mn}_{\mathrm{A}}$ & 3.34 & 6.0 & 0.016 & 7.33 \\
\hline $\mathrm{Mn}_{\mathrm{A}^{-}} \mathrm{Mn}_{\mathrm{A}}$ & 3.56 & 4.0 & 0.020 & 4.38 & & & & & \\
\hline
\end{tabular}

${ }^{a}$ Coordination numbers $(\mathrm{CN})$ were fixed.

${ }^{b}$ The value of the $R$-factor is $4.0 \%$ at Co K-edge and $2.6 \%$ at Mn K-edge, respectively. 
Table S7. Structural Parameters of $\mathrm{FeCo}_{2} \mathrm{O}_{4}$ Electrocatalysts Obtained from $\mathrm{Co}$ and $\mathrm{Fe}$ K-edge EXAFS Analysis

\begin{tabular}{|c|c|c|c|c|c|c|c|c|c|}
\hline \multicolumn{10}{|l|}{ Co K-edge } \\
\hline \multicolumn{5}{|c|}{ A site $\left(x_{\mathrm{A}}=0.42\right)$} & \multicolumn{5}{|c|}{$\mathrm{B}$ site $\left(x_{\mathrm{B}}=0.58\right)$} \\
\hline Bond & $r / \AA$ & $\mathrm{CN}$ & $\sigma^{2} / \AA^{2}$ & $\Delta E / \mathrm{eV}$ & Bond & $r / \AA$ & $\mathrm{CN}$ & $\overline{\sigma^{2} / \AA^{2}}$ & $\Delta E / \mathrm{eV}$ \\
\hline $\mathrm{Co}_{\mathrm{A}}-\mathrm{O}_{\mathrm{A}}$ & 1.83 & 4.0 & 0.004 & 10.95 & $\mathrm{Co}_{\mathrm{B}}-\mathrm{O}_{\mathrm{B}}$ & 1.93 & 6.0 & 0.003 & -2.15 \\
\hline $\mathrm{Co}_{\mathrm{A}^{-}} \mathrm{Co}_{\mathrm{B}}$ & 3.39 & 12.0 & 0.010 & 1.53 & $\mathrm{Co}_{\mathrm{B}}-\mathrm{Co}_{\mathrm{B}}$ & 2.87 & 6.0 & 0.008 & 1.53 \\
\hline $\mathrm{Co}_{\mathrm{A}}-\mathrm{O}_{\mathrm{B}}$ & 3.45 & 12.0 & 0.010 & -6.77 & $\mathrm{Co}_{\mathrm{B}}-\mathrm{Co}_{\mathrm{A}}$ & 3.39 & 6.0 & 0.010 & 1.53 \\
\hline $\mathrm{Co}_{\mathrm{A}^{-}} \mathrm{Co}_{\mathrm{A}}$ & 3.54 & 4.0 & 0.019 & 1.53 & & & & & \\
\hline \multicolumn{10}{|l|}{ Fe K-edge } \\
\hline \multicolumn{5}{|c|}{ A site $\left(x_{\mathrm{A}}=0.45\right)$} & \multicolumn{5}{|c|}{ B site $\left(x_{\mathrm{B}}=0.55\right)$} \\
\hline Bond & $r / \AA$ & $\mathrm{CN}$ & $\sigma^{2} / \AA^{2}$ & $\Delta E / \mathrm{eV}$ & Bond & $r / \AA$ & $\mathrm{CN}$ & $\sigma^{2} / \AA^{2}$ & $\Delta E / \mathrm{eV}$ \\
\hline$\overline{\mathrm{Fe}_{\mathrm{A}}-\mathrm{O}_{\mathrm{A}}}$ & 1.84 & 4.0 & 0.008 & 2.22 & $\mathrm{Fe}_{\mathrm{B}}-\mathrm{O}_{\mathrm{B}}$ & 1.96 & 6.0 & 0.005 & -4.04 \\
\hline $\mathrm{Fe}_{\mathrm{A}}-\mathrm{Fe}_{\mathrm{B}}$ & 3.42 & 12.0 & 0.020 & 8.00 & $\mathrm{Fe}_{\mathrm{B}}-\mathrm{Fe}_{\mathrm{B}}$ & 2.90 & 6.0 & 0.011 & -2.83 \\
\hline $\mathrm{Fe}_{\mathrm{A}}-\mathrm{O}_{\mathrm{B}}$ & 3.38 & 12.0 & 0.010 & 2.22 & $\mathrm{Fe}_{\mathrm{B}}-\mathrm{Fe}_{\mathrm{A}}$ & 3.42 & 6.0 & 0.020 & 8.00 \\
\hline $\mathrm{Fe}_{\mathrm{A}}-\mathrm{Fe}_{\mathrm{A}}$ & 3.42 & 4.0 & 0.011 & -10.00 & & & & & \\
\hline
\end{tabular}

${ }^{a}$ Coordination numbers $(\mathrm{CN})$ were fixed.

${ }^{b}$ The value of the $R$-factor is $1.0 \%$ at Co K-edge and $0.7 \%$ at Fe K-edge, respectively.

Table S8. Structural Parameters of $\mathrm{NiCo}_{2} \mathrm{O}_{4}$ Electrocatalysts Obtained from $\mathrm{Co}$ and $\mathrm{Ni}$ K-edge EXAFS Analysis

\begin{tabular}{|c|c|c|c|c|c|c|c|c|c|}
\hline \multicolumn{10}{|c|}{ Co K-edge } \\
\hline \multicolumn{5}{|c|}{ A site $\left(x_{\mathrm{A}}=0.05\right)$} & \multicolumn{5}{|c|}{$\mathrm{B}$ site $\left(x_{\mathrm{B}}=0.95\right)$} \\
\hline Bond & $r / \AA$ & $\mathrm{CN}$ & $\overline{\sigma^{2} / \AA^{2}}$ & $\Delta E / \mathrm{eV}$ & Bond & $r / \AA$ & $\overline{\mathrm{CN}}$ & $\overline{\sigma^{2} / \AA^{2}}$ & $\Delta E / \mathrm{eV}$ \\
\hline $\mathrm{Co}_{\mathrm{A}}-\mathrm{O}_{\mathrm{A}}$ & 1.84 & 4.0 & 0.012 & -0.29 & $\mathrm{Co}_{\mathrm{B}}-\mathrm{O}_{\mathrm{B}}$ & 1.92 & 6.0 & 0.003 & -0.29 \\
\hline $\mathrm{Co}_{\mathrm{A}^{-}} \mathrm{Co}_{\mathrm{B}}$ & 3.36 & 12.0 & 0.012 & 2.88 & $\mathrm{Co}_{\mathrm{B}}-\mathrm{Co}_{\mathrm{B}}$ & 2.87 & 6.0 & 0.005 & 2.88 \\
\hline $\mathrm{Co}_{\mathrm{A}}-\mathrm{O}_{\mathrm{B}}$ & 3.38 & 12.0 & 0.002 & -1.51 & $\mathrm{Co}_{\mathrm{B}}-\mathrm{Co}_{\mathrm{A}}$ & 3.36 & 6.0 & 0.012 & 2.88 \\
\hline $\mathrm{Co}_{\mathrm{A}^{-}} \mathrm{Co}_{\mathrm{A}}$ & 3.51 & 4.0 & 0.018 & 2.88 & & & & & \\
\hline \multicolumn{10}{|l|}{ Ni K-edge } \\
\hline \multicolumn{5}{|c|}{$\mathrm{A} \operatorname{site}\left(x_{\mathrm{A}}=0.15\right)$} & \multicolumn{5}{|c|}{$\mathrm{B}$ site $\left(x_{\mathrm{B}}=0.85\right)$} \\
\hline Bond & $r / \AA$ & $\mathrm{CN}$ & $\sigma^{2} / \AA^{2}$ & $\Delta E / \mathrm{eV}$ & Bond & $r / \AA$ & $\mathrm{CN}$ & $\sigma^{2} / \AA^{2}$ & $\Delta E / \mathrm{eV}$ \\
\hline$\overline{\mathrm{Ni}_{\mathrm{A}}-\mathrm{O}_{\mathrm{A}}}$ & 1.81 & 4.0 & 0.009 & -1.03 & $\mathrm{Ni}_{\mathrm{B}}-\mathrm{O}_{\mathrm{B}}$ & 1.98 & 6.0 & 0.007 & -2.10 \\
\hline $\mathrm{Ni}_{\mathrm{A}}-\mathrm{Ni}_{\mathrm{B}}$ & 3.34 & 12.0 & 0.020 & -0.02 & $\mathrm{Ni}_{\mathrm{B}}-\mathrm{Ni}_{\mathrm{B}}$ & 2.91 & 6.0 & 0.008 & -3.51 \\
\hline $\mathrm{Ni}_{\mathrm{A}}-\mathrm{O}_{\mathrm{B}}$ & 3.35 & 12.0 & 0.003 & -2.10 & $\mathrm{Ni}_{\mathrm{B}}-\mathrm{Ni}_{\mathrm{A}}$ & 3.34 & 6.0 & 0.020 & 12.45 \\
\hline $\mathrm{Ni}_{\mathrm{A}}-\mathrm{Ni}_{\mathrm{A}}$ & 3.48 & 4.0 & 0.003 & 12.45 & & & & & \\
\hline
\end{tabular}

${ }^{a}$ Coordination numbers $(\mathrm{CN})$ were fixed.

${ }^{b}$ The value of the $R$-factor is $1.9 \%$ at Co K-edge and $1.2 \%$ at Ni K-edge, respectively. 
Table S9. Structural Parameters of $\mathrm{ZnCo}_{2} \mathrm{O}_{4}$ Electrocatalysts Obtained from $\mathrm{Co}$ and $\mathrm{Zn}$ K-edge EXAFS Analysis

\begin{tabular}{|c|c|c|c|c|c|c|c|c|c|}
\hline \multicolumn{10}{|l|}{ Co K-edge } \\
\hline \multicolumn{5}{|c|}{ A site $\left(x_{\mathrm{A}}=1.0\right)$} & \multicolumn{5}{|c|}{$\mathrm{B}$ site $\left(x_{\mathrm{B}}=0.0\right)$} \\
\hline Bond & $r / \AA$ & $\mathrm{CN}$ & $\sigma^{2} / \AA^{2}$ & $\Delta E / \mathrm{eV}$ & Bond & $r / \AA$ & $\mathrm{CN}$ & $\overline{\sigma^{2} / \AA^{2}}$ & $\Delta E / \mathrm{eV}$ \\
\hline $\mathrm{Co}_{\mathrm{A}}-\mathrm{O}_{\mathrm{A}}$ & - & - & - & - & $\mathrm{Co}_{\mathrm{B}}-\mathrm{O}_{\mathrm{B}}$ & 1.93 & 6.0 & 0.008 & 2.27 \\
\hline $\mathrm{Co}_{\mathrm{A}^{-}} \mathrm{Co}_{\mathrm{B}}$ & - & - & - & - & $\mathrm{Co}_{\mathrm{B}}-\mathrm{Co}_{\mathrm{B}}$ & 2.87 & 6.0 & 0.009 & 2.10 \\
\hline $\mathrm{Co}_{\mathrm{A}}-\mathrm{O}_{\mathrm{B}}$ & - & - & - & - & $\mathrm{Co}_{\mathrm{B}}-\mathrm{Co}_{\mathrm{A}}$ & 3.39 & 6.0 & 0.009 & 2.10 \\
\hline $\mathrm{Co}_{\mathrm{A}^{-}} \mathrm{Co}_{\mathrm{A}}$ & - & - & - & - & & & & & \\
\hline \multicolumn{10}{|l|}{ Zn K-edge } \\
\hline \multicolumn{5}{|c|}{ A site $\left(x_{\mathrm{A}}=1.0\right)$} & \multicolumn{5}{|c|}{ B site $\left(x_{\mathrm{B}}=0.0\right)$} \\
\hline Bond & $r / \AA$ & $\mathrm{CN}$ & $\sigma^{2} / \AA^{2}$ & $\Delta E / \mathrm{eV}$ & Bond & $r / \AA$ & $\mathrm{CN}$ & $\sigma^{2} / \AA^{2}$ & $\Delta E / \mathrm{eV}$ \\
\hline $\mathrm{Zn}_{\mathrm{A}}-\mathrm{O}_{\mathrm{A}}$ & 1.99 & 4.0 & 0.006 & -0.36 & $\mathrm{Zn}_{\mathrm{B}}-\mathrm{O}_{\mathrm{B}}$ & - & - & - & - \\
\hline $\mathrm{Zn}_{\mathrm{A}}-\mathrm{Zn}_{\mathrm{B}}$ & 3.29 & 12.0 & 0.014 & 0.59 & $\mathrm{Zn}_{\mathrm{B}}-\mathrm{Zn}_{\mathrm{B}}$ & - & - & - & - \\
\hline $\mathrm{Zn}_{\mathrm{A}}-\mathrm{O}_{\mathrm{B}}$ & 3.38 & 12.0 & 0.024 & -10.5 & $\mathrm{Zn}_{\mathrm{B}}-\mathrm{Zn}_{\mathrm{A}}$ & - & - & - & - \\
\hline $\mathrm{Zn}_{\mathrm{A}}-\mathrm{Zn}_{\mathrm{A}}$ & 3.44 & 4.0 & 0.014 & 0.59 & & & & & \\
\hline
\end{tabular}

${ }^{a}$ Coordination numbers $(\mathrm{CN})$ were fixed.

${ }^{b}$ The value of the $R$-factor is $9.3 \%$ at Co K-edge and $3.1 \%$ at Zn K-edge, respectively. 
Table S10. Structural Parameters Obtained from Co K-edge EXAFS Analysis of $\mathrm{Co}_{3} \mathrm{O}_{4}$ Electrocatalysts Before and After the OER

Before EC $\quad(R$-factor: $1.4 \%)$

\begin{tabular}{|lcccc|ccccc|}
\hline \multicolumn{5}{c|}{ A site } & \multicolumn{5}{c|}{ B site } \\
\hline Bond & $r / \AA$ & $\mathrm{CN}$ & $\sigma^{2} / \AA^{2}$ & $\Delta E / \mathrm{eV}$ & Bond & $r / \AA$ & $\mathrm{CN}$ & $\sigma^{2} / \AA^{2}$ & $\Delta E / \mathrm{eV}$ \\
\hline $\mathrm{Co}_{\mathrm{A}}-\mathrm{O}_{\mathrm{A}}$ & 1.86 & 4.0 & 0.003 & 6.44 & $\mathrm{Co}_{\mathrm{B}}-\mathrm{O}_{\mathrm{B}}$ & 1.92 & 6.0 & 0.004 & -10.00 \\
$\mathrm{Co}_{\mathrm{A}}-\mathrm{Co}_{\mathrm{B}}$ & 3.43 & 12.0 & 0.007 & 9.49 & $\mathrm{Co}_{\mathrm{B}}-\mathrm{Co}_{\mathrm{B}}$ & 2.90 & 6.0 & 0.005 & 9.49 \\
$\mathrm{Co}_{\mathrm{A}}-\mathrm{O}_{\mathrm{B}}$ & 3.58 & 12.0 & 0.006 & 6.44 & $\mathrm{Co}_{\mathrm{B}}-\mathrm{Co}_{\mathrm{A}}$ & 3.43 & 6.0 & 0.007 & 9.49 \\
$\mathrm{Co}_{\mathrm{A}}-\mathrm{Co}_{\mathrm{A}}$ & 3.64 & 4.0 & 0.003 & 9.49 & & & & & \\
\hline
\end{tabular}

After CV $\quad(R$-factor: $1.3 \%)$

\begin{tabular}{|lcccc|ccccc|}
\hline \multicolumn{5}{c|}{ A site } & \multicolumn{5}{c|}{ B site } \\
\hline Bond & $r / \AA$ & $\mathrm{CN}$ & $\sigma^{2} / \AA^{2}$ & $\Delta E / \mathrm{eV}$ & Bond & $r / \AA$ & $\mathrm{CN}$ & $\sigma^{2} / \AA^{2}$ & $\Delta E / \mathrm{eV}$ \\
\hline $\mathrm{Co}_{\mathrm{A}}-\mathrm{O}_{\mathrm{A}}$ & 1.86 & 4.0 & 0.004 & 6.55 & $\mathrm{Co}_{\mathrm{B}}-\mathrm{O}_{\mathrm{B}}$ & 1.92 & 6.0 & 0.003 & -10.00 \\
$\mathrm{Co}_{\mathrm{A}}-\mathrm{Co}_{\mathrm{B}}$ & 3.43 & 12.0 & 0.008 & 9.30 & $\mathrm{Co}_{\mathrm{B}}-\mathrm{Co}_{\mathrm{B}}$ & 2.90 & 6.0 & 0.006 & 9.30 \\
$\mathrm{Co}_{\mathrm{A}}-\mathrm{O}_{\mathrm{B}}$ & 3.57 & 12.0 & 0.005 & 6.55 & $\mathrm{Co}_{\mathrm{B}}-\mathrm{Co}_{\mathrm{A}}$ & 3.43 & 6.0 & 0.008 & 9.30 \\
$\mathrm{Co}_{\mathrm{A}}-\mathrm{Co}_{\mathrm{A}}$ & 3.65 & 4.0 & 0.006 & 9.30 & & & & & \\
\hline
\end{tabular}

After CA $\quad(R$-factor: $3.4 \%)$

\begin{tabular}{|lcccc|ccccc|}
\hline \multicolumn{5}{c|}{ A site } & \multicolumn{5}{c|}{ B site } \\
\hline Bond & $r / \AA$ & $\mathrm{CN}$ & $\sigma^{2} / \AA^{2}$ & $\Delta E / \mathrm{eV}$ & Bond & $r / \AA$ & $\mathrm{CN}$ & $\sigma^{2} / \AA^{2}$ & $\Delta E / \mathrm{eV}$ \\
\hline $\mathrm{Co}_{\mathrm{A}}-\mathrm{O}_{\mathrm{A}}$ & 1.86 & 4.0 & 0.005 & 6.39 & $\mathrm{Co}_{\mathrm{B}}-\mathrm{O}_{\mathrm{B}}$ & 1.92 & 6.0 & 0.004 & -9.78 \\
$\mathrm{Co}_{\mathrm{A}}-\mathrm{Co}_{\mathrm{B}}$ & 3.43 & 12.0 & 0.010 & 9.30 & $\mathrm{Co}_{\mathrm{B}}-\mathrm{Co}_{\mathrm{B}}$ & 2.90 & 6.0 & 0.005 & 9.30 \\
$\mathrm{Co}_{\mathrm{A}}-\mathrm{O}_{\mathrm{B}}$ & 3.57 & 12.0 & 0.008 & 6.39 & $\mathrm{Co}_{\mathrm{B}}-\mathrm{Co}_{\mathrm{A}}$ & 3.43 & 6.0 & 0.010 & 9.30 \\
$\mathrm{Co}_{\mathrm{A}}-\mathrm{Co}_{\mathrm{A}}$ & 3.66 & 4.0 & 0.007 & 9.30 & & & & & \\
\hline
\end{tabular}


Table S11. Structural Parameters Obtained from Co and Mn K-edge EXAFS Analysis of $\mathrm{MnCo}_{2} \mathrm{O}_{4}$ Electrocatalysts Before and After the OER

Before EC

\begin{tabular}{|c|c|c|c|c|c|c|c|c|c|}
\hline \multicolumn{10}{|c|}{ Co K-edge $\quad(R$-factor: $2.5 \%)$} \\
\hline \multicolumn{5}{|c|}{ A site $\left(x_{\mathrm{A}}=0.46\right)$} & \multicolumn{5}{|c|}{$\mathrm{B}$ site $\left(x_{\mathrm{B}}=0.54\right)$} \\
\hline Bond & $r / \AA$ & $\mathrm{CN}$ & $\overline{\sigma^{2} / \AA^{2}}$ & $\Delta E / \mathrm{eV}$ & Bond & $r / \AA$ & $\mathrm{CN}$ & $\sigma^{2} / \AA^{2}$ & $\Delta E / \mathrm{eV}$ \\
\hline $\mathrm{Co}_{\mathrm{A}}-\mathrm{O}_{\mathrm{A}}$ & 1.82 & 4.0 & 0.007 & 10.00 & $\mathrm{Co}_{\mathrm{B}}-\mathrm{O}_{\mathrm{B}}$ & 1.92 & 6.0 & 0.005 & -3.06 \\
\hline $\mathrm{Co}_{\mathrm{A}^{-}}-\mathrm{Co}_{\mathrm{B}}$ & 3.35 & 12.0 & 0.011 & -4.07 & $\mathrm{Co}_{\mathrm{B}}-\mathrm{Co}_{\mathrm{B}}$ & 2.86 & 6.0 & 0.009 & 1.79 \\
\hline $\mathrm{Co}_{\mathrm{A}}-\mathrm{O}_{\mathrm{B}}$ & 3.36 & 12.0 & 0.007 & -10.00 & $\mathrm{Co}_{\mathrm{B}}-\mathrm{Co}_{\mathrm{A}}$ & 3.35 & 6.0 & 0.011 & 1.79 \\
\hline $\mathrm{Co}_{\mathrm{A}^{-}}-\mathrm{Co}_{\mathrm{A}}$ & 3.43 & 4.0 & 0.007 & 1.79 & & & & & \\
\hline \multicolumn{10}{|c|}{ Mn K-edge $\quad(R$-factor: $1.1 \%)$} \\
\hline \multicolumn{5}{|c|}{ A site $\left(x_{\mathrm{A}}=0.58\right)$} & \multicolumn{5}{|c|}{$\mathrm{B}$ site $\left(x_{\mathrm{B}}=0.42\right)$} \\
\hline Bond & $r / \AA$ & $\mathrm{CN}$ & $\overline{\sigma^{2} / \AA^{2}}$ & $\Delta E / \mathrm{eV}$ & Bond & $r / \AA$ & $\mathrm{CN}$ & $\sigma^{2} / \AA^{2}$ & $\Delta E / \mathrm{eV}$ \\
\hline $\mathrm{Mn}_{\mathrm{A}}-\mathrm{O}_{\mathrm{A}}$ & 1.88 & 4.0 & 0.003 & 3.41 & $\mathrm{Mn}_{\mathrm{B}}-\mathrm{O}_{\mathrm{B}}$ & 2.03 & 6.0 & 0.020 & -8.17 \\
\hline $\mathrm{Mn}_{\mathrm{A}^{-}} \mathrm{Mn}_{\mathrm{B}}$ & 3.30 & 12.0 & 0.014 & 1.96 & $\mathrm{Mn}_{\mathrm{B}}-\mathrm{Mn}_{\mathrm{B}}$ & 2.81 & 6.0 & 0.010 & -9.49 \\
\hline $\mathrm{Mn}_{\mathrm{A}}-\mathrm{O}_{\mathrm{B}}$ & 3.42 & 12.0 & 0.003 & -0.39 & $\mathrm{Mn}_{\mathrm{B}}-\mathrm{Mn}_{\mathrm{A}}$ & 3.30 & 6.0 & 0.014 & 1.96 \\
\hline $\mathrm{Mn}_{\mathrm{A}}-\mathrm{Mn}_{\mathrm{A}}$ & 3.55 & 4.0 & 0.020 & 3.41 & & & & & \\
\hline
\end{tabular}

\section{After CV}

\begin{tabular}{|c|c|c|c|c|c|c|c|c|c|}
\hline \multicolumn{10}{|c|}{ Co K-edge $\quad(R$-factor: $3.0 \%)$} \\
\hline \multicolumn{5}{|c|}{ A site $\left(x_{\mathrm{A}}=0.56\right)$} & \multicolumn{5}{|c|}{$\mathrm{B}$ site $\left(x_{\mathrm{B}}=0.44\right)$} \\
\hline Bond & $r / \AA$ & $\mathrm{CN}$ & $\overline{\sigma^{2} / \AA^{2}}$ & $\Delta E / \mathrm{eV}$ & Bond & $r / \AA$ & $\mathrm{CN}$ & $\overline{\sigma^{2} / \AA^{2}}$ & $\Delta E / \mathrm{eV}$ \\
\hline $\mathrm{Co}_{\mathrm{A}}-\mathrm{O}_{\mathrm{A}}$ & 1.74 & 4.0 & 0.015 & 16.76 & $\mathrm{Co}_{\mathrm{B}}-\mathrm{O}_{\mathrm{B}}$ & 1.92 & 6.0 & 0.006 & 1.21 \\
\hline $\mathrm{Co}_{A^{-}}-\mathrm{Co}_{\mathrm{B}}$ & 3.36 & 12.0 & 0.013 & 0.63 & $\mathrm{Co}_{\mathrm{B}^{-}} \mathrm{Co}_{\mathrm{B}}$ & 2.86 & 6.0 & 0.011 & 0.63 \\
\hline $\mathrm{Co}_{\mathrm{A}}-\mathrm{O}_{\mathrm{B}}$ & 3.28 & 12.0 & 0.015 & 19.28 & $\mathrm{Co}_{\mathrm{B}}-\mathrm{Co}_{\mathrm{A}}$ & 3.36 & 6.0 & 0.013 & 0.63 \\
\hline $\mathrm{Co}_{\mathrm{A}^{-}}-\mathrm{Co}_{\mathrm{A}}$ & 3.41 & 4.0 & 0.003 & 0.63 & & & & & \\
\hline \multicolumn{10}{|c|}{$(R$-factor: $4.6 \%)$} \\
\hline \multicolumn{5}{|c|}{ A site $\left(x_{\mathrm{A}}=0.64\right)$} & \multicolumn{5}{|c|}{ B site $\left(x_{\mathrm{B}}=0.36\right)$} \\
\hline Bond & $\overline{r / \AA}$ & $\mathrm{CN}$ & $\overline{\sigma^{2} / \AA^{2}}$ & $\Delta E / \mathrm{eV}$ & Bond & $r / \AA$ & $\mathrm{CN}$ & $\overline{\sigma^{2} / \AA^{2}}$ & $\Delta E / \mathrm{eV}$ \\
\hline $\mathrm{Mn}_{\mathrm{A}}-\mathrm{O}_{\mathrm{A}}$ & 1.88 & 4.0 & 0.003 & -0.45 & $\mathrm{Mn}_{\mathrm{B}}-\mathrm{O}_{\mathrm{B}}$ & 2.03 & 6.0 & 0.004 & -21.39 \\
\hline $\mathrm{Mn}_{\mathrm{A}^{-}}-\mathrm{Mn}_{\mathrm{B}}$ & 3.29 & 12.0 & 0.019 & -3.11 & $\mathrm{Mn}_{\mathrm{B}}-\mathrm{Mn}_{\mathrm{B}}$ & 2.89 & 6.0 & 0.008 & 4.84 \\
\hline $\mathrm{Mn}_{\mathrm{A}}-\mathrm{O}_{\mathrm{B}}$ & 3.42 & 12.0 & 0.007 & -5.83 & $\mathrm{Mn}_{\mathrm{B}}-\mathrm{Mn}_{\mathrm{A}}$ & 3.29 & 6.0 & 0.019 & -3.11 \\
\hline $\mathrm{Mn}_{\mathrm{A}}-\mathrm{Mn}_{\mathrm{A}}$ & 3.55 & 4.0 & 0.045 & -0.45 & & & & & \\
\hline
\end{tabular}


After CA

\begin{tabular}{|c|c|c|c|c|c|c|c|c|c|}
\hline \multicolumn{10}{|c|}{ Co K-edge $\quad(R$-factor: $3.9 \%)$} \\
\hline \multicolumn{5}{|c|}{ A site $\left(x_{\mathrm{A}}=0.65\right)$} & \multicolumn{5}{|c|}{$\mathrm{B}$ site $\left(x_{\mathrm{B}}=0.35\right)$} \\
\hline Bond & $r / \AA$ & $\mathrm{CN}$ & $\overline{\sigma^{2} / \AA^{2}}$ & $\Delta E / \mathrm{eV}$ & Bond & $r / \AA$ & $\overline{\mathrm{CN}}$ & $\sigma^{2} / \AA^{2}$ & $\Delta E / \mathrm{eV}$ \\
\hline $\mathrm{Co}_{\mathrm{A}}-\mathrm{O}_{\mathrm{A}}$ & 1.95 & 4.0 & 0.003 & 18.62 & $\mathrm{Co}_{\mathrm{B}}-\mathrm{O}_{\mathrm{B}}$ & 1.92 & 6.0 & 0.012 & -2.20 \\
\hline $\mathrm{Co}_{A^{-}}-\mathrm{Co}_{\mathrm{B}}$ & 3.42 & 12.0 & 0.015 & 6.73 & $\mathrm{Co}_{\mathrm{B}}-\mathrm{Co}_{\mathrm{B}}$ & 2.92 & 6.0 & 0.008 & 6.73 \\
\hline $\mathrm{Co}_{\mathrm{A}}-\mathrm{O}_{\mathrm{B}}$ & 3.49 & 12.0 & 0.003 & 2.32 & $\mathrm{Co}_{\mathrm{B}}-\mathrm{Co}_{\mathrm{A}}$ & 3.42 & 6.0 & 0.015 & 6.73 \\
\hline $\mathrm{Co}_{\mathrm{A}^{-}} \mathrm{Co}_{\mathrm{A}}$ & 3.44 & 4.0 & 0.003 & 6.73 & & & & & \\
\hline \multicolumn{10}{|c|}{$(R$-factor: $2.9 \%)$} \\
\hline \multicolumn{5}{|c|}{ A site $\left(x_{\mathrm{A}}=0.75\right)$} & \multicolumn{5}{|c|}{$\mathrm{B}$ site $\left(x_{\mathrm{B}}=0.25\right)$} \\
\hline Bond & $r / \AA$ & $\mathrm{CN}$ & $\overline{\sigma^{2} / \AA^{2}}$ & $\Delta E / \mathrm{eV}$ & Bond & $r / \AA$ & $\mathrm{CN}$ & $\sigma^{2} / \AA^{2}$ & $\Delta E / \mathrm{eV}$ \\
\hline $\mathrm{Mn}_{\mathrm{A}}-\mathrm{O}_{\mathrm{A}}$ & 1.98 & 4.0 & 0.009 & 2.68 & $\mathrm{Mn}_{\mathrm{B}}-\mathrm{O}_{\mathrm{B}}$ & 2.13 & 6.0 & -0.002 & -15.89 \\
\hline $\mathrm{Mn}_{\mathrm{A}^{-}} \mathrm{Mn}_{\mathrm{B}}$ & 3.05 & 12.0 & 0.019 & -39.40 & $\mathrm{Mn}_{\mathrm{B}}-\mathrm{Mn}_{\mathrm{B}}$ & 2.18 & 6.0 & 0.567 & -99.79 \\
\hline $\mathrm{Mn}_{\mathrm{A}}-\mathrm{O}_{\mathrm{B}}$ & 3.52 & 12.0 & 0.011 & -21.54 & $\mathrm{Mn}_{\mathrm{B}}-\mathrm{Mn}_{\mathrm{A}}$ & 3.05 & 6.0 & 0.019 & -39.40 \\
\hline $\mathrm{Mn}_{\mathrm{A}}-\mathrm{Mn}_{\mathrm{A}}$ & 3.65 & 4.0 & 8.500 & 2.68 & & & & & \\
\hline
\end{tabular}


Table S12. Structural Parameters Obtained from Co and Fe K-edge EXAFS Analysis of $\mathrm{FeCo}_{2} \mathrm{O}_{4}$ Electrocatalysts Before and After the OER

Before EC

\begin{tabular}{|c|c|c|c|c|c|c|c|c|c|}
\hline \multicolumn{10}{|c|}{ Co K-edge $\quad(R$-factor: $1.1 \%)$} \\
\hline \multicolumn{5}{|c|}{ A site $\left(x_{\mathrm{A}}=0.48\right)$} & \multicolumn{5}{|c|}{$\mathrm{B}$ site $\left(x_{\mathrm{B}}=0.52\right)$} \\
\hline Bond & $r / \AA$ & $\mathrm{CN}$ & $\overline{\sigma^{2} / \AA^{2}}$ & $\Delta E / \mathrm{eV}$ & Bond & $r / \AA$ & $\mathrm{CN}$ & $\overline{\sigma^{2} / \AA^{2}}$ & $\Delta E / \mathrm{eV}$ \\
\hline $\mathrm{Co}_{\mathrm{A}}-\mathrm{O}_{\mathrm{A}}$ & 1.76 & 4.0 & 0.003 & 9.37 & $\mathrm{Co}_{\mathrm{B}}-\mathrm{O}_{\mathrm{B}}$ & 1.91 & 6.0 & 0.003 & -4.23 \\
\hline $\mathrm{Co}_{\mathrm{A}^{-}}-\mathrm{Co}_{\mathrm{B}}$ & 3.43 & 12.0 & 0.010 & 4.38 & $\mathrm{Co}_{\mathrm{B}}-\mathrm{Co}_{\mathrm{B}}$ & 2.91 & 6.0 & 0.008 & 4.38 \\
\hline $\mathrm{Co}_{\mathrm{A}}-\mathrm{O}_{\mathrm{B}}$ & 3.43 & 12.0 & 0.003 & -6.77 & $\mathrm{Co}_{\mathrm{B}}-\mathrm{Co}_{\mathrm{A}}$ & 3.43 & 6.0 & 0.010 & 4.38 \\
\hline $\mathrm{Co}_{\mathrm{A}^{-}} \mathrm{Co}_{\mathrm{A}}$ & 3.67 & 4.0 & 0.003 & 4.38 & & & & & \\
\hline \multicolumn{10}{|c|}{$(R$-factor: $0.5 \%)$} \\
\hline \multicolumn{5}{|c|}{ A site $\left(x_{\mathrm{A}}=0.53\right)$} & \multicolumn{5}{|c|}{$\mathrm{B}$ site $\left(x_{\mathrm{B}}=0.47\right)$} \\
\hline Bond & $r / \AA$ & $\mathrm{CN}$ & $\sigma^{2} / \AA^{2}$ & $\Delta E / \mathrm{eV}$ & Bond & $r / \AA$ & $\mathrm{CN}$ & $\sigma^{2} / \AA^{2}$ & $\Delta E / \mathrm{eV}$ \\
\hline $\mathrm{Fe}_{\mathrm{A}}-\mathrm{O}_{\mathrm{A}}$ & 1.91 & 4.0 & 0.006 & 4.35 & $\mathrm{Fe}_{\mathrm{B}}-\mathrm{O}_{\mathrm{B}}$ & 1.98 & 6.0 & 0.003 & -5.20 \\
\hline $\mathrm{Fe}_{\mathrm{A}^{-}}-\mathrm{Fe}_{\mathrm{B}}$ & 3.36 & 12.0 & 0.013 & -5.83 & $\mathrm{Fe}_{\mathrm{B}}-\mathrm{Fe}_{\mathrm{B}}$ & 2.86 & 6.0 & 0.008 & -10.00 \\
\hline $\mathrm{Fe}_{\mathrm{A}}-\mathrm{O}_{\mathrm{B}}$ & 3.45 & 12.0 & 0.020 & -3.23 & $\mathrm{Fe}_{\mathrm{B}}-\mathrm{Fe}_{\mathrm{A}}$ & 3.36 & 6.0 & 0.013 & -5.83 \\
\hline $\mathrm{Fe}_{\mathrm{A}}-\mathrm{Fe}_{\mathrm{A}}$ & 3.81 & 4.0 & 0.009 & -10.00 & & & & & \\
\hline
\end{tabular}

\section{After CV}

\begin{tabular}{|c|c|c|c|c|c|c|c|c|c|}
\hline \multicolumn{10}{|c|}{ Co K-edge $\quad(R$-factor: $1.3 \%)$} \\
\hline \multicolumn{5}{|c|}{ A site $\left(x_{\mathrm{A}}=0.46\right)$} & \multicolumn{5}{|c|}{$\mathrm{B}$ site $\left(x_{\mathrm{B}}=0.54\right)$} \\
\hline Bond & $r / \AA$ & $\mathrm{CN}$ & $\overline{\sigma^{2} / \AA^{2}}$ & $\Delta E / \mathrm{eV}$ & Bond & $r / \AA$ & $\mathrm{CN}$ & $\overline{\sigma^{2} / \AA^{2}}$ & $\Delta E / \mathrm{eV}$ \\
\hline $\mathrm{Co}_{\mathrm{A}}-\mathrm{O}_{\mathrm{A}}$ & 1.76 & 4.0 & 0.004 & 8.02 & $\mathrm{Co}_{\mathrm{B}}-\mathrm{O}_{\mathrm{B}}$ & 1.91 & 6.0 & 0.004 & -4.50 \\
\hline $\mathrm{Co}_{A^{-}}-\mathrm{Co}_{\mathrm{B}}$ & 3.42 & 12.0 & 0.011 & 3.87 & $\mathrm{Co}_{\mathrm{B}}-\mathrm{Co}_{\mathrm{B}}$ & 2.91 & 6.0 & 0.009 & 3.87 \\
\hline $\mathrm{Co}_{\mathrm{A}}-\mathrm{O}_{\mathrm{B}}$ & 3.42 & 12.0 & 0.003 & -6.77 & $\mathrm{Co}_{\mathrm{B}}-\mathrm{Co}_{\mathrm{A}}$ & 3.42 & 6.0 & 0.011 & 3.87 \\
\hline $\mathrm{Co}_{\mathrm{A}^{-}}-\mathrm{Co}_{\mathrm{A}}$ & 3.67 & 4.0 & 0.004 & 3.87 & & & & & \\
\hline \multicolumn{10}{|c|}{ (R-factor: $1.3 \%)$} \\
\hline \multicolumn{5}{|c|}{ A site $\left(x_{\mathrm{A}}=0.54\right)$} & \multicolumn{5}{|c|}{$\mathrm{B}$ site $\left(x_{\mathrm{B}}=0.46\right)$} \\
\hline Bond & $r / \AA$ & $\mathrm{CN}$ & $\overline{\sigma^{2} / \AA^{2}}$ & $\Delta E / \mathrm{eV}$ & Bond & $r / \AA$ & $\mathrm{CN}$ & $\overline{\sigma^{2} / \AA^{2}}$ & $\Delta E / \mathrm{eV}$ \\
\hline $\mathrm{Fe}_{\mathrm{A}}-\mathrm{O}_{\mathrm{A}}$ & 1.90 & 4.0 & 0.005 & 3.34 & $\mathrm{Fe}_{\mathrm{B}}-\mathrm{O}_{\mathrm{B}}$ & 2.06 & 6.0 & 0.003 & 0.39 \\
\hline $\mathrm{Fe}_{\mathrm{A}}-\mathrm{Fe}_{\mathrm{B}}$ & 3.34 & 12.0 & 0.012 & -6.01 & $\mathrm{Fe}_{\mathrm{B}}-\mathrm{Fe}_{\mathrm{B}}$ & 2.88 & 6.0 & 0.011 & -10.06 \\
\hline $\mathrm{Fe}_{\mathrm{A}}-\mathrm{O}_{\mathrm{B}}$ & 3.44 & 12.0 & 0.012 & -2.79 & $\mathrm{Fe}_{\mathrm{B}}-\mathrm{Fe}_{\mathrm{A}}$ & 3.34 & 6.0 & 0.012 & -6.01 \\
\hline $\mathrm{Fe}_{\mathrm{A}}-\mathrm{Fe}_{\mathrm{A}}$ & 3.82 & 4.0 & 0.003 & -10.00 & & & & & \\
\hline
\end{tabular}


After CA

\begin{tabular}{|c|c|c|c|c|c|c|c|c|c|}
\hline \multicolumn{10}{|c|}{ Co K-edge $\quad(R$-factor: $1.8 \%)$} \\
\hline \multicolumn{5}{|c|}{ A site $\left(x_{\mathrm{A}}=0.47\right)$} & \multicolumn{5}{|c|}{$\mathrm{B}$ site $\left(x_{\mathrm{B}}=0.53\right)$} \\
\hline Bond & $r / \AA$ & $\mathrm{CN}$ & $\overline{\sigma^{2} / \AA^{2}}$ & $\Delta E / \mathrm{eV}$ & Bond & $r / \AA$ & $\mathrm{CN}$ & $\sigma^{2} / \AA^{2}$ & $\Delta E / \mathrm{eV}$ \\
\hline $\mathrm{Co}_{\mathrm{A}}-\mathrm{O}_{\mathrm{A}}$ & 1.74 & 4.0 & 0.003 & 10.00 & $\mathrm{Co}_{\mathrm{B}}-\mathrm{O}_{\mathrm{B}}$ & 1.91 & 6.0 & 0.003 & -3.38 \\
\hline $\mathrm{Co}_{\mathrm{A}^{-}} \mathrm{Co}_{\mathrm{B}}$ & 3.42 & 12.0 & 0.010 & 4.70 & $\mathrm{Co}_{\mathrm{B}}-\mathrm{Co}_{\mathrm{B}}$ & 2.91 & 6.0 & 0.007 & 4.70 \\
\hline $\mathrm{Co}_{\mathrm{A}}-\mathrm{O}_{\mathrm{B}}$ & 3.42 & 12.0 & 0.003 & -6.77 & $\mathrm{Co}_{\mathrm{B}}-\mathrm{Co}_{\mathrm{A}}$ & 3.42 & 6.0 & 0.010 & 4.70 \\
\hline $\mathrm{Co}_{\mathrm{A}^{-}}-\mathrm{Co}_{\mathrm{A}}$ & 3.67 & 4.0 & 0.003 & 4.70 & & & & & \\
\hline \multicolumn{10}{|c|}{$(R$-factor: $0.6 \%)$} \\
\hline \multicolumn{5}{|c|}{ A site $\left(x_{\mathrm{A}}=0.56\right)$} & \multicolumn{5}{|c|}{ B site $\left(x_{\mathrm{B}}=0.44\right)$} \\
\hline Bond & $r / \AA$ & $\mathrm{CN}$ & $\overline{\sigma^{2} / \AA^{2}}$ & $\Delta E / \mathrm{eV}$ & Bond & $r / \AA$ & $\mathrm{CN}$ & $\sigma^{2} / \AA^{2}$ & $\Delta E / \mathrm{eV}$ \\
\hline $\mathrm{Fe}_{\mathrm{A}}-\mathrm{O}_{\mathrm{A}}$ & 1.92 & 4.0 & 0.006 & 4.35 & $\mathrm{Fe}_{\mathrm{B}}-\mathrm{O}_{\mathrm{B}}$ & 2.04 & 6.0 & 0.003 & 1.62 \\
\hline $\mathrm{Fe}_{\mathrm{A}}-\mathrm{Fe}_{\mathrm{B}}$ & 3.35 & 12.0 & 0.013 & -5.83 & $\mathrm{Fe}_{\mathrm{B}}-\mathrm{Fe}_{\mathrm{B}}$ & 2.87 & 6.0 & 0.008 & -10.00 \\
\hline $\mathrm{Fe}_{\mathrm{A}}-\mathrm{O}_{\mathrm{B}}$ & 3.46 & 12.0 & 0.020 & -3.23 & $\mathrm{Fe}_{\mathrm{B}}-\mathrm{Fe}_{\mathrm{A}}$ & 3.35 & 6.0 & 0.013 & -5.83 \\
\hline $\mathrm{Fe}_{\mathrm{A}}-\mathrm{Fe}_{\mathrm{A}}$ & 3.82 & 4.0 & 0.009 & -10.00 & & & & & \\
\hline
\end{tabular}


Table S13. Structural Parameters Obtained from Co and Ni K-edge EXAFS Analysis of $\mathrm{NiCo}_{2} \mathrm{O}_{4}$ Electrocatalysts Before and After the OER

Before EC

\begin{tabular}{|c|c|c|c|c|c|c|c|c|c|}
\hline \multicolumn{10}{|c|}{ Co K-edge $\quad(R$-factor: $3.1 \%)$} \\
\hline \multicolumn{5}{|c|}{ A site $\left(x_{\mathrm{A}}=0.11\right)$} & \multicolumn{5}{|c|}{$\mathrm{B}$ site $\left(x_{\mathrm{B}}=0.89\right)$} \\
\hline Bond & $r / \AA$ & $\mathrm{CN}$ & $\overline{\sigma^{2} / \AA^{2}}$ & $\Delta E / \mathrm{eV}$ & Bond & $r / \AA$ & $\mathrm{CN}$ & $\sigma^{2} / \AA^{2}$ & $\Delta E / \mathrm{eV}$ \\
\hline $\mathrm{Co}_{\mathrm{A}}-\mathrm{O}_{\mathrm{A}}$ & 1.72 & 4.0 & 0.008 & -7.43 & $\mathrm{Co}_{\mathrm{B}}-\mathrm{O}_{\mathrm{B}}$ & 1.90 & 6.0 & 0.003 & -7.43 \\
\hline $\mathrm{Co}_{\mathrm{A}^{-}}-\mathrm{Co}_{\mathrm{B}}$ & 3.44 & 12.0 & 0.009 & 7.27 & $\mathrm{Co}_{\mathrm{B}}-\mathrm{Co}_{\mathrm{B}}$ & 2.91 & 6.0 & 0.008 & 7.27 \\
\hline $\mathrm{Co}_{\mathrm{A}}-\mathrm{O}_{\mathrm{B}}$ & 3.54 & 12.0 & 0.005 & 3.10 & $\mathrm{Co}_{\mathrm{B}}-\mathrm{Co}_{\mathrm{A}}$ & 3.44 & 6.0 & 0.009 & 7.27 \\
\hline $\mathrm{Co}_{\mathrm{A}^{-}} \mathrm{Co}_{\mathrm{A}}$ & 3.67 & 4.0 & 0.003 & 7.27 & & & & & \\
\hline \multicolumn{10}{|c|}{$(R$-factor: $3.5 \%)$} \\
\hline \multicolumn{5}{|c|}{ A site $\left(x_{\mathrm{A}}=0.18\right)$} & \multicolumn{5}{|c|}{$\mathrm{B}$ site $\left(x_{\mathrm{B}}=0.82\right)$} \\
\hline Bond & $r / \AA$ & $\mathrm{CN}$ & $\sigma^{2} / \AA^{2}$ & $\Delta E / \mathrm{eV}$ & Bond & $r / \AA$ & $\mathrm{CN}$ & $\sigma^{2} / \AA^{2}$ & $\Delta E / \mathrm{eV}$ \\
\hline $\mathrm{Ni}_{\mathrm{A}}-\mathrm{O}_{\mathrm{A}}$ & 1.63 & 4.0 & 0.003 & 7.59 & $\mathrm{Ni}_{\mathrm{B}}-\mathrm{O}_{\mathrm{B}}$ & 2.03 & 6.0 & 0.012 & 3.65 \\
\hline $\mathrm{Ni}_{\mathrm{A}}-\mathrm{Ni}_{\mathrm{B}}$ & 3.35 & 12.0 & 0.014 & -3.82 & $\mathrm{Ni}_{\mathrm{B}}-\mathrm{Ni}_{\mathrm{B}}$ & 2.88 & 6.0 & 0.008 & -6.03 \\
\hline $\mathrm{Ni}_{\mathrm{A}}-\mathrm{O}_{\mathrm{B}}$ & 3.17 & 12.0 & 0.013 & -10.0 & $\mathrm{Ni}_{\mathrm{B}}-\mathrm{Ni}_{\mathrm{A}}$ & 3.35 & 6.0 & 0.014 & -3.82 \\
\hline $\mathrm{Ni}_{\mathrm{A}}-\mathrm{Ni}_{\mathrm{A}}$ & 3.31 & 4.0 & 0.020 & 7.59 & & & & & \\
\hline
\end{tabular}

\section{After CV}

\begin{tabular}{|c|c|c|c|c|c|c|c|c|c|}
\hline \multicolumn{10}{|c|}{ Co K-edge $\quad(R$-factor: $1.9 \%)$} \\
\hline \multicolumn{5}{|c|}{ A site $\left(x_{\mathrm{A}}=0.11\right)$} & \multicolumn{5}{|c|}{$\mathrm{B}$ site $\left(x_{\mathrm{B}}=0.89\right)$} \\
\hline Bond & $r / \AA$ & $\mathrm{CN}$ & $\overline{\sigma^{2} / \AA^{2}}$ & $\Delta E / \mathrm{eV}$ & Bond & $r / \AA$ & $\mathrm{CN}$ & $\overline{\sigma^{2} / \AA^{2}}$ & $\Delta E / \mathrm{eV}$ \\
\hline $\mathrm{Co}_{\mathrm{A}}-\mathrm{O}_{\mathrm{A}}$ & 1.52 & 4.0 & 0.014 & -7.57 & $\mathrm{Co}_{\mathrm{B}}-\mathrm{O}_{\mathrm{B}}$ & 1.86 & 6.0 & 0.005 & -7.57 \\
\hline $\mathrm{Co}_{\mathrm{A}^{-}} \mathrm{Co}_{\mathrm{B}}$ & 3.48 & 12.0 & 0.014 & 7.41 & $\mathrm{Co}_{\mathrm{B}^{-}} \mathrm{Co}_{\mathrm{B}}$ & 2.93 & 6.0 & 0.007 & 7.41 \\
\hline $\mathrm{Co}_{\mathrm{A}}-\mathrm{O}_{\mathrm{B}}$ & 3.59 & 12.0 & 0.019 & 2.63 & $\mathrm{Co}_{\mathrm{B}}-\mathrm{Co}_{\mathrm{A}}$ & 3.48 & 6.0 & 0.014 & 7.41 \\
\hline $\mathrm{Co}_{\mathrm{A}^{-}}-\mathrm{Co}_{\mathrm{A}}$ & 3.73 & 4.0 & 0.004 & 7.41 & & & & & \\
\hline \multicolumn{10}{|c|}{ (R-factor: $8.7 \%)$} \\
\hline \multicolumn{5}{|c|}{ A site $\left(x_{\mathrm{A}}=0.31\right)$} & \multicolumn{5}{|c|}{ B site $\left(x_{\mathrm{B}}=0.69\right)$} \\
\hline Bond & $r / \AA$ & $\mathrm{CN}$ & $\overline{\sigma^{2} / \AA^{2}}$ & $\Delta E / \mathrm{eV}$ & Bond & $r / \AA$ & $\mathrm{CN}$ & $\overline{\sigma^{2} / \AA^{2}}$ & $\Delta E / \mathrm{eV}$ \\
\hline $\mathrm{Ni}_{\mathrm{A}}-\mathrm{O}_{\mathrm{A}}$ & 1.63 & 4.0 & 0.003 & -12.48 & $\mathrm{Ni}_{\mathrm{B}}-\mathrm{O}_{\mathrm{B}}$ & 1.99 & 6.0 & 0.004 & -3.91 \\
\hline $\mathrm{Ni}_{\mathrm{A}}-\mathrm{Ni}_{\mathrm{B}}$ & 3.39 & 12.0 & 0.019 & 1.63 & $\mathrm{Ni}_{\mathrm{B}}-\mathrm{Ni}_{\mathrm{B}}$ & 2.90 & 6.0 & 0.008 & -5.79 \\
\hline $\mathrm{Ni}_{\mathrm{A}}-\mathrm{O}_{\mathrm{B}}$ & 3.17 & 12.0 & 0.012 & -11.29 & $\mathrm{Ni}_{\mathrm{B}}-\mathrm{Ni}_{\mathrm{A}}$ & 3.39 & 6.0 & 0.019 & 1.63 \\
\hline $\mathrm{Ni}_{\mathrm{A}}-\mathrm{Ni}_{\mathrm{A}}$ & 3.30 & 4.0 & 0.041 & -12.48 & & & & & \\
\hline
\end{tabular}


After CA

\begin{tabular}{|c|c|c|c|c|c|c|c|c|c|}
\hline \multicolumn{10}{|c|}{ Co K-edge $\quad(R$-factor: $3.5 \%)$} \\
\hline \multicolumn{5}{|c|}{ A site $\left(x_{\mathrm{A}}=0.11\right)$} & \multicolumn{5}{|c|}{$\mathrm{B}$ site $\left(x_{\mathrm{B}}=0.89\right)$} \\
\hline Bond & $r / \AA$ & $\mathrm{CN}$ & $\sigma^{2} / \AA^{2}$ & $\Delta E / \mathrm{eV}$ & Bond & $r / \AA$ & $\mathrm{CN}$ & $\overline{\sigma^{2} / \AA^{2}}$ & $\Delta E / \mathrm{eV}$ \\
\hline $\mathrm{Co}_{\mathrm{A}}-\mathrm{O}_{\mathrm{A}}$ & 1.63 & 4.0 & 0.020 & -7.26 & $\mathrm{Co}_{\mathrm{B}}-\mathrm{O}_{\mathrm{B}}$ & 1.88 & 6.0 & 0.005 & -7.26 \\
\hline $\mathrm{Co}_{\mathrm{A}^{-}}-\mathrm{Co}_{\mathrm{B}}$ & 3.45 & 12.0 & 0.010 & 7.09 & $\mathrm{Co}_{\mathrm{B}}-\mathrm{Co}_{\mathrm{B}}$ & 2.92 & 6.0 & 0.008 & 7.09 \\
\hline $\mathrm{Co}_{\mathrm{A}}-\mathrm{O}_{\mathrm{B}}$ & 3.55 & 12.0 & 0.004 & 2.36 & $\mathrm{Co}_{\mathrm{B}}-\mathrm{Co}_{\mathrm{A}}$ & 3.45 & 6.0 & 0.010 & 7.09 \\
\hline $\mathrm{Co}_{\mathrm{A}}-\mathrm{Co}_{\mathrm{A}}$ & 3.69 & 4.0 & 0.002 & 7.09 & & & & & \\
\hline \multicolumn{10}{|c|}{$(R$-factor: $0.2 \%)$} \\
\hline \multicolumn{5}{|c|}{ A site $\left(x_{\mathrm{A}}=0.32\right)$} & \multicolumn{5}{|c|}{$\mathrm{B}$ site $\left(x_{\mathrm{B}}=0.68\right)$} \\
\hline Bond & $r / \AA$ & $\mathrm{CN}$ & $\sigma^{2} / \AA^{2}$ & $\Delta E / \mathrm{eV}$ & Bond & $r / \AA$ & $\mathrm{CN}$ & $\sigma^{2} / \AA^{2}$ & $\Delta E / \mathrm{eV}$ \\
\hline $\mathrm{Ni}_{\mathrm{A}}-\mathrm{O}_{\mathrm{A}}$ & 1.71 & 4.0 & 0.023 & 18.43 & $\mathrm{Ni}_{\mathrm{B}}-\mathrm{O}_{\mathrm{B}}$ & 2.03 & 6.0 & 0.005 & 4.37 \\
\hline $\mathrm{Ni}_{\mathrm{A}}-\mathrm{Ni}_{\mathrm{B}}$ & 3.50 & 12.0 & 0.023 & 10.74 & $\mathrm{Ni}_{\mathrm{B}}-\mathrm{Ni}_{\mathrm{B}}$ & 2.90 & 6.0 & 0.012 & -4.54 \\
\hline $\mathrm{Ni}_{\mathrm{A}}-\mathrm{O}_{\mathrm{B}}$ & 3.25 & 12.0 & 0.003 & -7.40 & $\mathrm{Ni}_{\mathrm{B}}-\mathrm{Ni}_{\mathrm{A}}$ & 3.50 & 6.0 & 0.023 & 10.74 \\
\hline $\mathrm{Ni}_{\mathrm{A}}-\mathrm{Ni}_{\mathrm{A}}$ & 3.38 & 4.0 & 0.030 & 18.43 & & & & & \\
\hline
\end{tabular}


Table S14. Structural Parameters Obtained from Co and Zn K-edge EXAFS Analysis of $\mathrm{ZnCo}_{2} \mathrm{O}_{4}$ Electrocatalysts Before and After the OER

Before EC

\begin{tabular}{|c|c|c|c|c|c|c|c|c|c|}
\hline \multicolumn{10}{|c|}{ Co K-edge $\quad(R$-factor: $6.4 \%)$} \\
\hline \multicolumn{5}{|c|}{ A site $\left(x_{\mathrm{A}}=1.0\right)$} & \multicolumn{5}{|c|}{$\mathrm{B}$ site $\left(x_{\mathrm{B}}=0.0\right)$} \\
\hline Bond & $r / \AA$ & $\mathrm{CN}$ & $\sigma^{2} / \AA^{2}$ & $\Delta E / \mathrm{eV}$ & Bond & $r / \AA$ & $\overline{\mathrm{CN}}$ & $\sigma^{2} / \AA^{2}$ & $\Delta E / \mathrm{eV}$ \\
\hline $\mathrm{Co}_{\mathrm{A}}-\mathrm{O}_{\mathrm{A}}$ & - & - & - & - & $\mathrm{Co}_{\mathrm{B}}-\mathrm{O}_{\mathrm{B}}$ & 1.91 & 6.0 & 0.006 & -0.44 \\
\hline $\mathrm{Co}_{\mathrm{A}^{-}}-\mathrm{Co}_{\mathrm{B}}$ & - & - & - & - & $\mathrm{Co}_{\mathrm{B}}-\mathrm{Co}_{\mathrm{B}}$ & 2.87 & 6.0 & 0.008 & 0.73 \\
\hline $\mathrm{Co}_{\mathrm{A}}-\mathrm{O}_{\mathrm{B}}$ & - & - & - & - & $\mathrm{Co}_{\mathrm{B}}-\mathrm{Co}_{\mathrm{A}}$ & 3.39 & 6.0 & 0.009 & 0.73 \\
\hline $\mathrm{Co}_{\mathrm{A}^{-}}-\mathrm{Co}_{\mathrm{A}}$ & - & - & - & - & & & & & \\
\hline \multicolumn{10}{|c|}{ (R-factor: $3.6 \%)$} \\
\hline \multicolumn{5}{|c|}{ A site $\left(x_{\mathrm{A}}=1.0\right)$} & \multicolumn{5}{|c|}{$\mathrm{B}$ site $\left(x_{\mathrm{B}}=0.0\right)$} \\
\hline Bond & $r / \AA$ & $\mathrm{CN}$ & $\sigma^{2} / \AA^{2}$ & $\Delta E / \mathrm{eV}$ & Bond & $r / \AA$ & $\mathrm{CN}$ & $\sigma^{2} / \AA^{2}$ & $\Delta E / \mathrm{eV}$ \\
\hline $\mathrm{Zn}_{\mathrm{A}}-\mathrm{O}_{\mathrm{A}}$ & 1.95 & 4.0 & 0.004 & -9.51 & $\mathrm{Zn}_{\mathrm{B}}-\mathrm{O}_{\mathrm{B}}$ & - & - & - & - \\
\hline $\mathrm{Zn}_{\mathrm{A}}-\mathrm{Zn}_{\mathrm{B}}$ & 3.40 & 12.0 & 0.013 & 4.31 & $\mathrm{Zn}_{\mathrm{B}}-\mathrm{Zn}_{\mathrm{B}}$ & - & - & - & - \\
\hline $\mathrm{Zn}_{\mathrm{A}}-\mathrm{O}_{\mathrm{B}}$ & 3.28 & 12.0 & 0.020 & -9.61 & $\mathrm{Zn}_{\mathrm{B}}-\mathrm{Zn}_{\mathrm{A}}$ & - & - & - & - \\
\hline $\mathrm{Zn}_{\mathrm{A}}-\mathrm{Zn}_{\mathrm{A}}$ & 3.55 & 4.0 & 0.013 & 4.31 & & & & & \\
\hline
\end{tabular}

\section{After CV}

\begin{tabular}{|c|c|c|c|c|c|c|c|c|c|}
\hline \multicolumn{10}{|c|}{ Co K-edge $\quad(R$-factor: $4.9 \%)$} \\
\hline \multicolumn{5}{|c|}{ A site $\left(x_{\mathrm{A}}=1.0\right)$} & \multicolumn{5}{|c|}{$\mathrm{B}$ site $\left(x_{\mathrm{B}}=0.0\right)$} \\
\hline Bond & $r / \AA$ & $\mathrm{CN}$ & $\overline{\sigma^{2} / \AA^{2}}$ & $\Delta E / \mathrm{eV}$ & Bond & $r / \AA$ & $\mathrm{CN}$ & $\sigma^{2} / \AA^{2}$ & $\Delta E / \mathrm{eV}$ \\
\hline $\mathrm{Co}_{\mathrm{A}}-\mathrm{O}_{\mathrm{A}}$ & - & - & - & - & $\mathrm{Co}_{\mathrm{B}}-\mathrm{O}_{\mathrm{B}}$ & 1.93 & 6.0 & 0.006 & -3.52 \\
\hline $\mathrm{Co}_{A^{-}}-\mathrm{Co}_{\mathrm{B}}$ & - & - & - & - & $\mathrm{Co}_{\mathrm{B}}-\mathrm{Co}_{\mathrm{B}}$ & 2.88 & 6.0 & 0.007 & -5.77 \\
\hline $\mathrm{Co}_{\mathrm{A}}-\mathrm{O}_{\mathrm{B}}$ & - & - & - & - & $\mathrm{Co}_{\mathrm{B}}-\mathrm{Co}_{\mathrm{A}}$ & 3.40 & 6.0 & 0.009 & -5.77 \\
\hline $\mathrm{Co}_{\mathrm{A}^{-}}-\mathrm{Co}_{\mathrm{A}}$ & - & - & - & - & & & & & \\
\hline \multicolumn{10}{|c|}{ (R-factor: $4.8 \%)$} \\
\hline \multicolumn{5}{|c|}{ A site $\left(x_{\mathrm{A}}=1.0\right)$} & \multicolumn{5}{|c|}{$\mathrm{B}$ site $\left(x_{\mathrm{B}}=0.0\right)$} \\
\hline Bond & $r / \AA$ & $\mathrm{CN}$ & $\overline{\sigma^{2} / \AA^{2}}$ & $\Delta E / \mathrm{eV}$ & Bond & $r / \AA$ & $\mathrm{CN}$ & $\sigma^{2} / \AA^{2}$ & $\Delta E / \mathrm{eV}$ \\
\hline $\mathrm{Zn}_{\mathrm{A}}-\mathrm{O}_{\mathrm{A}}$ & 1.94 & 4.0 & 0.003 & -11.89 & $\mathrm{Zn}_{\mathrm{B}}-\mathrm{O}_{\mathrm{B}}$ & - & - & - & - \\
\hline $\mathrm{Zn}_{\mathrm{A}}-\mathrm{Zn}_{\mathrm{B}}$ & 3.40 & 12.0 & 0.011 & 3.91 & $\mathrm{Zn}_{\mathrm{B}}-\mathrm{Zn}_{\mathrm{B}}$ & - & - & - & - \\
\hline $\mathrm{Zn}_{\mathrm{A}}-\mathrm{O}_{\mathrm{B}}$ & 3.28 & 12.0 & 0.021 & -9.23 & $\mathrm{Zn}_{\mathrm{B}}-\mathrm{Zn}_{\mathrm{A}}$ & - & - & - & - \\
\hline $\mathrm{Zn}_{\mathrm{A}^{-}} \mathrm{Zn}_{\mathrm{A}}$ & 3.55 & 4.0 & 0.011 & 3.91 & & & & & \\
\hline
\end{tabular}


After CA

\begin{tabular}{|c|c|c|c|c|c|c|c|c|c|}
\hline \multicolumn{10}{|c|}{ Co K-edge $\quad(R$-factor: $4.8 \%)$} \\
\hline \multicolumn{5}{|c|}{ A site $\left(x_{\mathrm{A}}=1.0\right)$} & \multicolumn{5}{|c|}{$\mathrm{B}$ site $\left(x_{\mathrm{B}}=0.0\right)$} \\
\hline Bond & $r / \AA$ & $\mathrm{CN}$ & $\sigma^{2} / \AA^{2}$ & $\Delta E / \mathrm{eV}$ & Bond & $r / \AA$ & $\mathrm{CN}$ & $\sigma^{2} / \AA^{2}$ & $\Delta E / \mathrm{eV}$ \\
\hline $\mathrm{Co}_{\mathrm{A}}-\mathrm{O}_{\mathrm{A}}$ & - & - & - & - & $\mathrm{Co}_{\mathrm{B}}-\mathrm{O}_{\mathrm{B}}$ & 1.90 & 6.0 & 0.007 & -8.32 \\
\hline $\mathrm{Co}_{\mathrm{A}}-\mathrm{Co}_{\mathrm{B}}$ & - & - & - & - & $\mathrm{Co}_{\mathrm{B}}-\mathrm{Co}_{\mathrm{B}}$ & 2.89 & 6.0 & 0.008 & -3.71 \\
\hline $\mathrm{Co}_{\mathrm{A}}-\mathrm{O}_{\mathrm{B}}$ & - & - & - & - & $\mathrm{Co}_{\mathrm{B}}-\mathrm{Co}_{\mathrm{A}}$ & 3.42 & 6.0 & 0.009 & -3.71 \\
\hline $\mathrm{Co}_{A^{-}}-\mathrm{Co}_{\mathrm{A}}$ & - & - & - & - & & & & & \\
\hline \multicolumn{10}{|c|}{$(R$-factor: $2.4 \%)$} \\
\hline \multicolumn{5}{|c|}{ A site $\left(x_{\mathrm{A}}=1.0\right)$} & \multicolumn{5}{|c|}{$\mathrm{B}$ site $\left(x_{\mathrm{B}}=0.0\right)$} \\
\hline Bond & $r / \AA$ & $\mathrm{CN}$ & $\sigma^{2} / \AA^{2}$ & $\Delta E / \mathrm{eV}$ & Bond & $r / \AA$ & $\mathrm{CN}$ & $\sigma^{2} / \AA^{2}$ & $\Delta E / \mathrm{eV}$ \\
\hline $\mathrm{Zn}_{\mathrm{A}}-\mathrm{O}_{\mathrm{A}}$ & 2.00 & 4.0 & 0.006 & -2.55 & $\mathrm{Zn}_{\mathrm{B}}-\mathrm{O}_{\mathrm{B}}$ & - & - & - & - \\
\hline $\mathrm{Zn}_{\mathrm{A}^{-}} \mathrm{Zn}_{\mathrm{B}}$ & 3.39 & 12.0 & 0.012 & 3.63 & $\mathrm{Zn}_{\mathrm{B}}-\mathrm{Zn}_{\mathrm{B}}$ & - & - & - & - \\
\hline $\mathrm{Zn}_{\mathrm{A}}-\mathrm{O}_{\mathrm{B}}$ & 3.40 & 12.0 & 0.020 & -2.00 & $\mathrm{Zn}_{\mathrm{B}}-\mathrm{Zn}_{\mathrm{A}}$ & - & - & - & - \\
\hline $\mathrm{Zn}_{\mathrm{A}}-\mathrm{Zn}_{\mathrm{A}}$ & 3.54 & 4.0 & 0.012 & 3.63 & & & & & \\
\hline
\end{tabular}


(a)

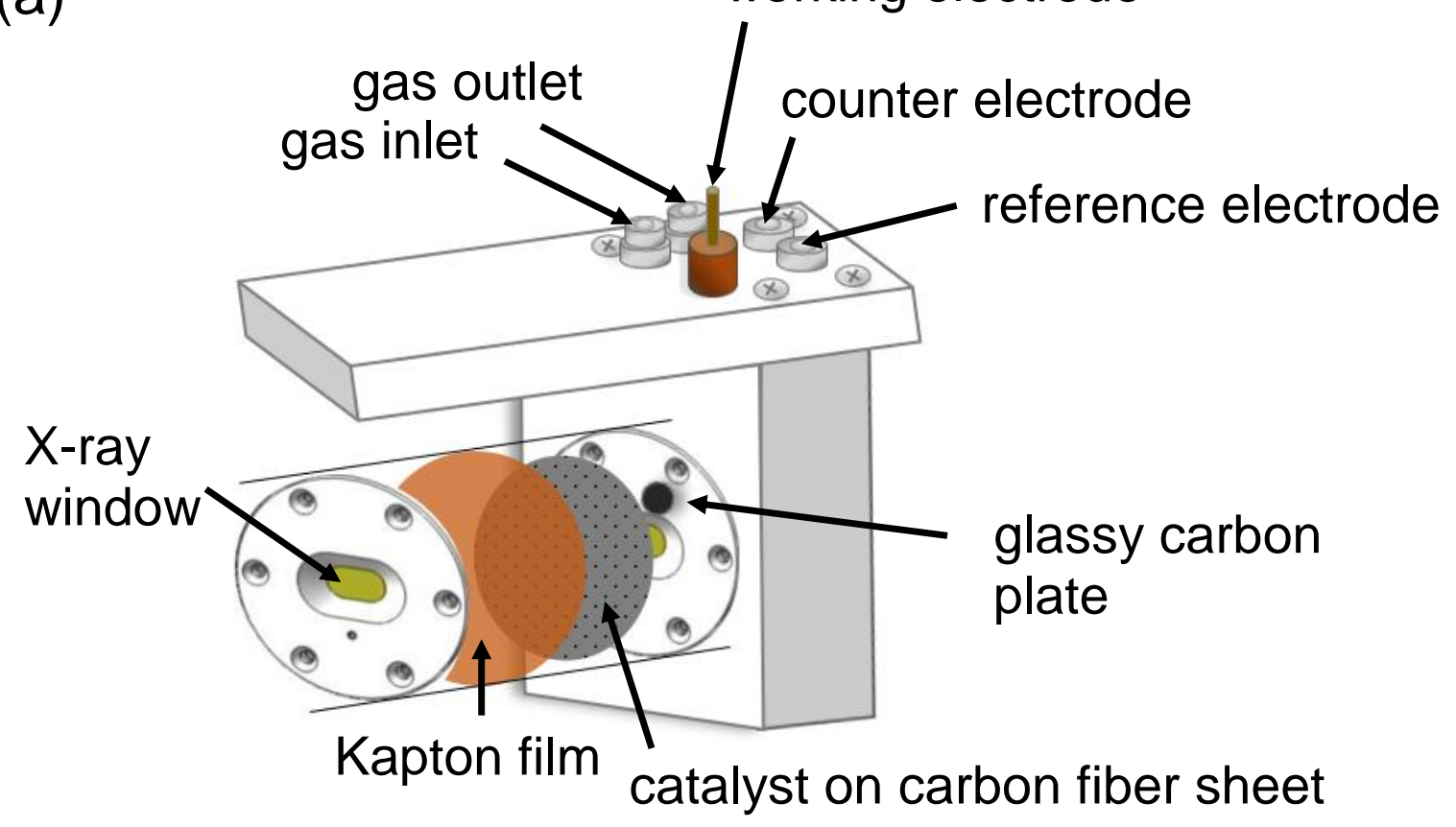

(b)
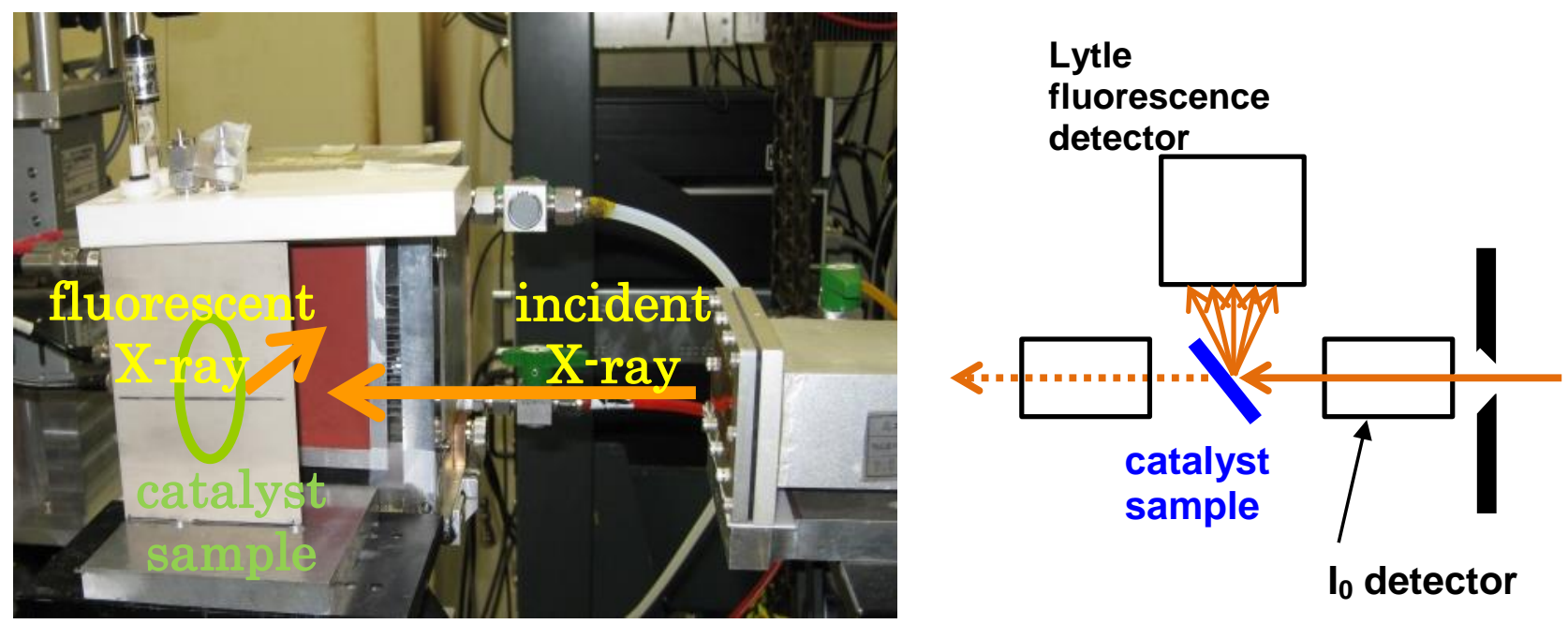

Figure S1. (a) Schematic illustration of the in situ electrochemical XAFS cell for fluorescence data collection. A three-electrode configuration cell was used for the electrochemical characterization. (b) Side and top-down view showing paths of X-rays and locations of sample, $\mathrm{I}_{0}$ ionization detector, and the Lytle fluorescence detector. 

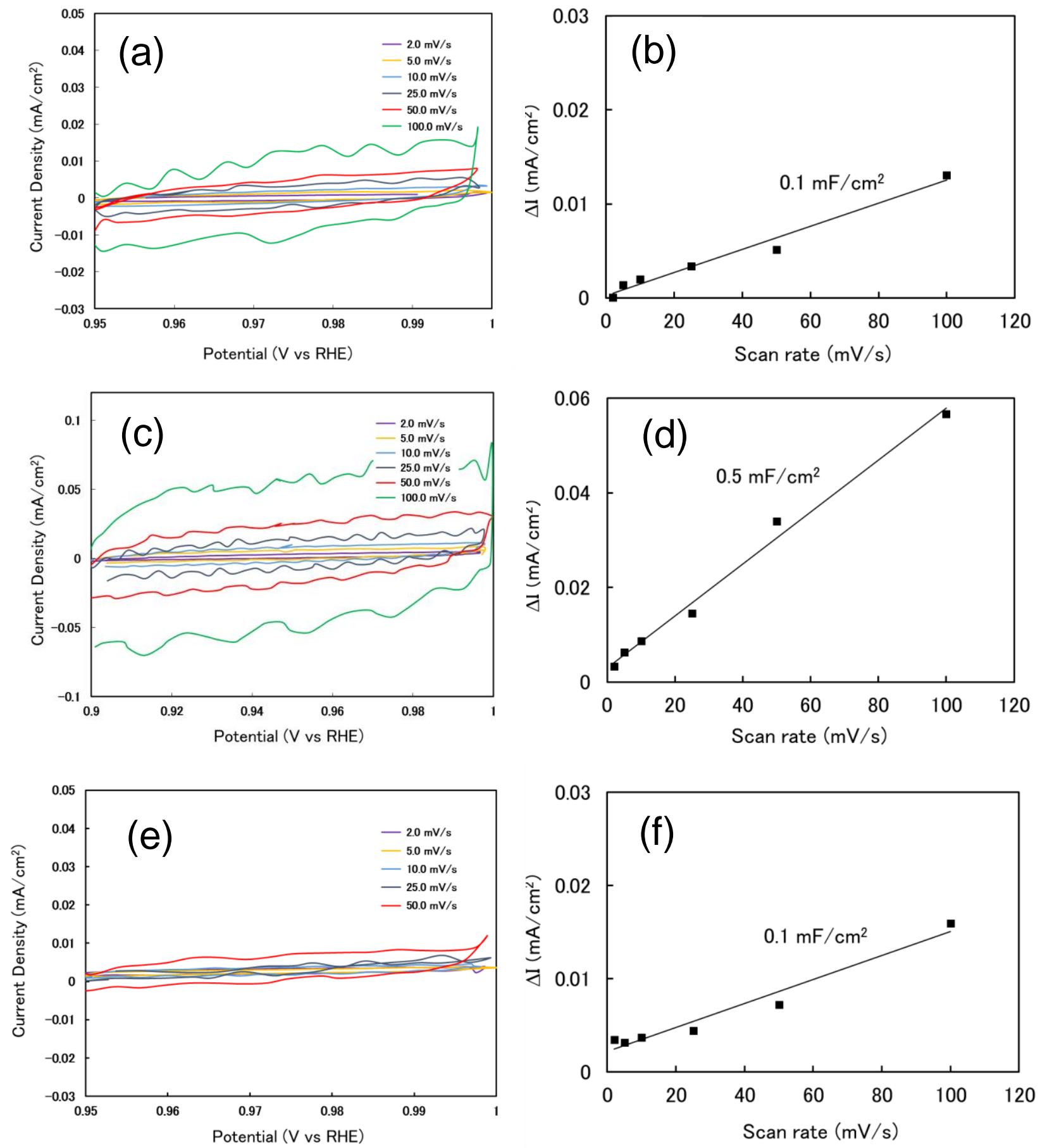

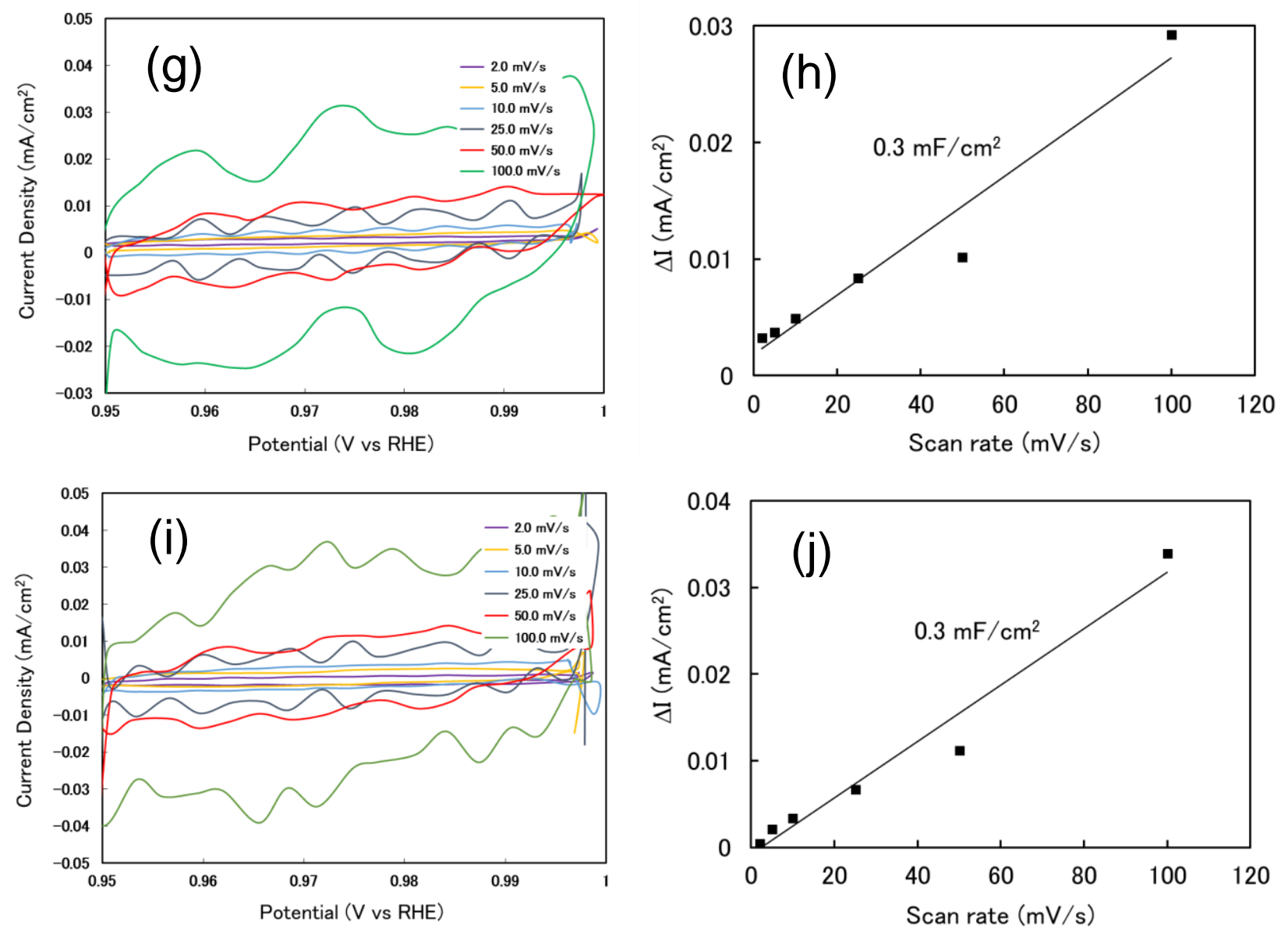

Figure S2. Double-layer capacitance measurements for determining the electrochemically active surface area (ECSA) performed in $0.1 \mathrm{M} \mathrm{KOH}$ solution. Cyclic voltammetry curves at various scan rates of (a) $\mathrm{Co}_{3} \mathrm{O}_{4}$, (c) $\mathrm{MnCo}_{2} \mathrm{O}_{4}$, (e) $\mathrm{FeCo}_{2} \mathrm{O}_{4}$, (g) $\mathrm{NiCo}_{2} \mathrm{O}_{4}$, and (i) $\mathrm{ZnCo}_{2} \mathrm{O}_{4}$ catalyst. The capacitive current densities at $0.975 \mathrm{~V}$ (vs. RHE) (at $0.95 \mathrm{~V}$ in the case of $\mathrm{MnCo}_{2} \mathrm{O}_{4}$ ) are plotted as a function of scan for (b) $\mathrm{Co}_{3} \mathrm{O}_{4}$, (d) $\mathrm{MnCo}_{2} \mathrm{O}_{4}$, (f) $\mathrm{FeCo}_{2} \mathrm{O}_{4}$, (h) $\mathrm{NiCo}_{2} \mathrm{O}_{4}$, and (j) $\mathrm{ZnCo}_{2} \mathrm{O}_{4}$ catalyst. 
(a)
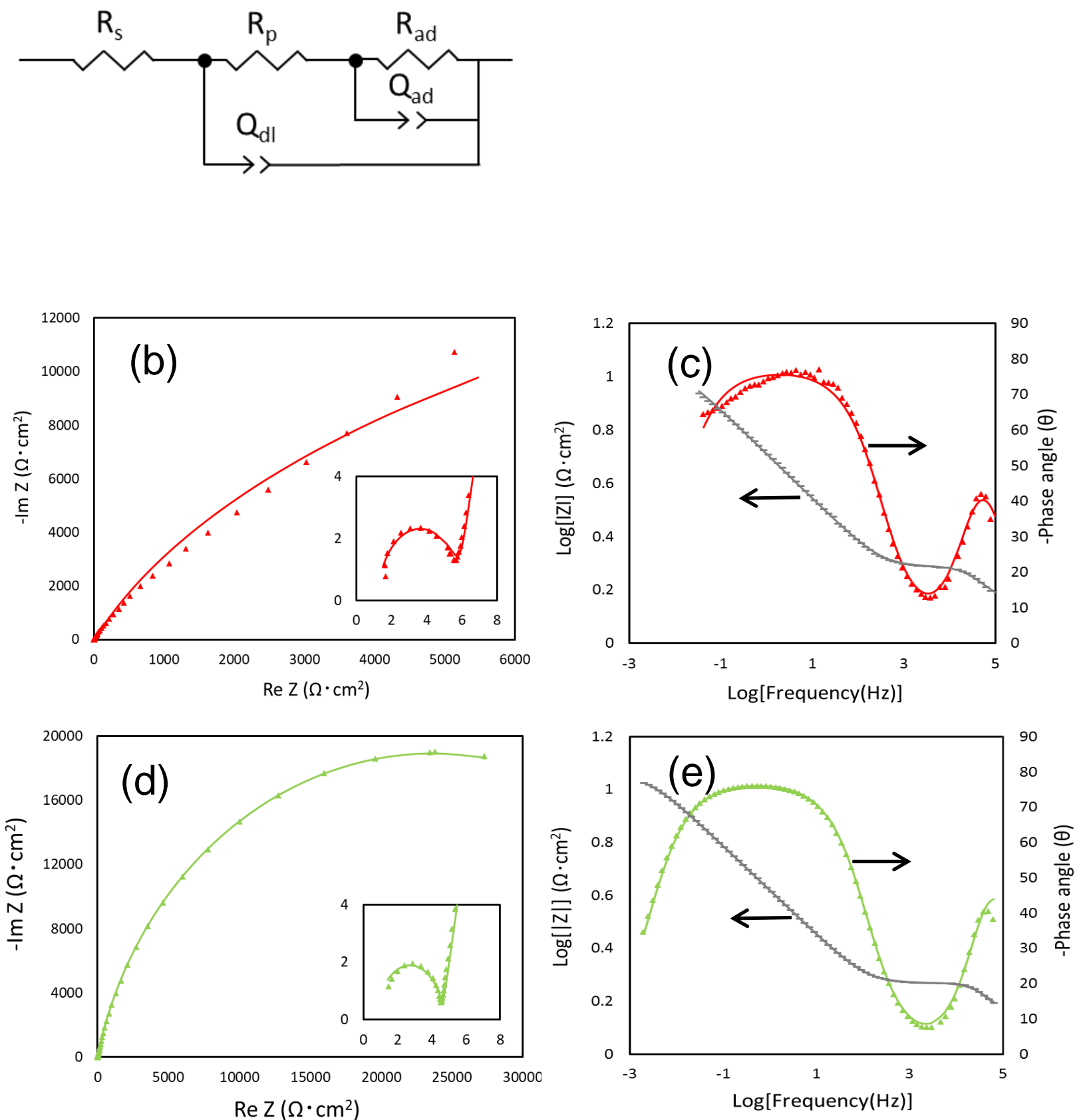

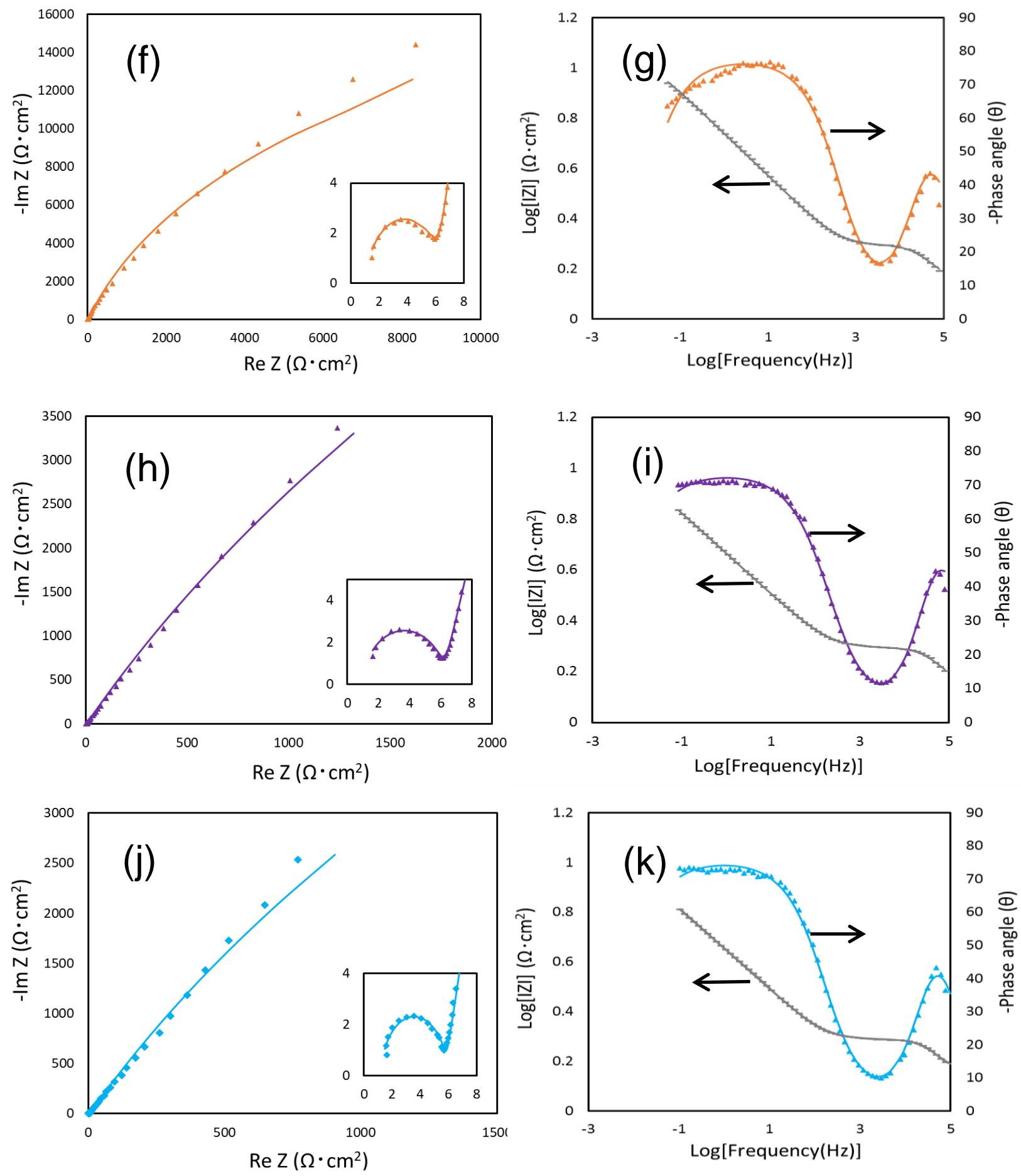

Figure S3. (a) Equivalent circuit used to fit the experimental data of electrochemical impedance spectroscopy (EIS). Nyquist and Bode plots of the electrochemical impedance spectra for various $\mathrm{MCo}_{2} \mathrm{O}_{4}(\mathrm{M}=\mathrm{Mn}, \mathrm{Fe}, \mathrm{Co}, \mathrm{Ni}$, and $\mathrm{Zn})$ catalysts in $0.1 \mathrm{M} \mathrm{KOH}$ electrolyte at $1.0 \mathrm{~V}$ (vs. RHE). Experimental (markers) and simulated (full lines) Nyquist plots of (b) $\mathrm{Co}_{3} \mathrm{O}_{4}$, (d) $\mathrm{MnCo}_{2} \mathrm{O}_{4}$, (f) $\mathrm{FeCo}_{2} \mathrm{O}_{4}$, (h) $\mathrm{NiCo}_{2} \mathrm{O}_{4}$, and (j) $\mathrm{ZnCo}_{2} \mathrm{O}_{4}$ catalyst, and Bode plots of (c) $\mathrm{Co}_{3} \mathrm{O}_{4}$, (e) $\mathrm{MnCo}_{2} \mathrm{O}_{4}$, (g) $\mathrm{FeCo}_{2} \mathrm{O}_{4}$, (i) $\mathrm{NiCo}_{2} \mathrm{O}_{4}$, and (k) $\mathrm{ZnCo}_{2} \mathrm{O}_{4}$ catalyst. Range of investigated frequency is $100 \mathrm{kHz}-$ $0.1 \mathrm{~Hz}$. The high frequency region of the Nyquist plot is shown as an inset. 

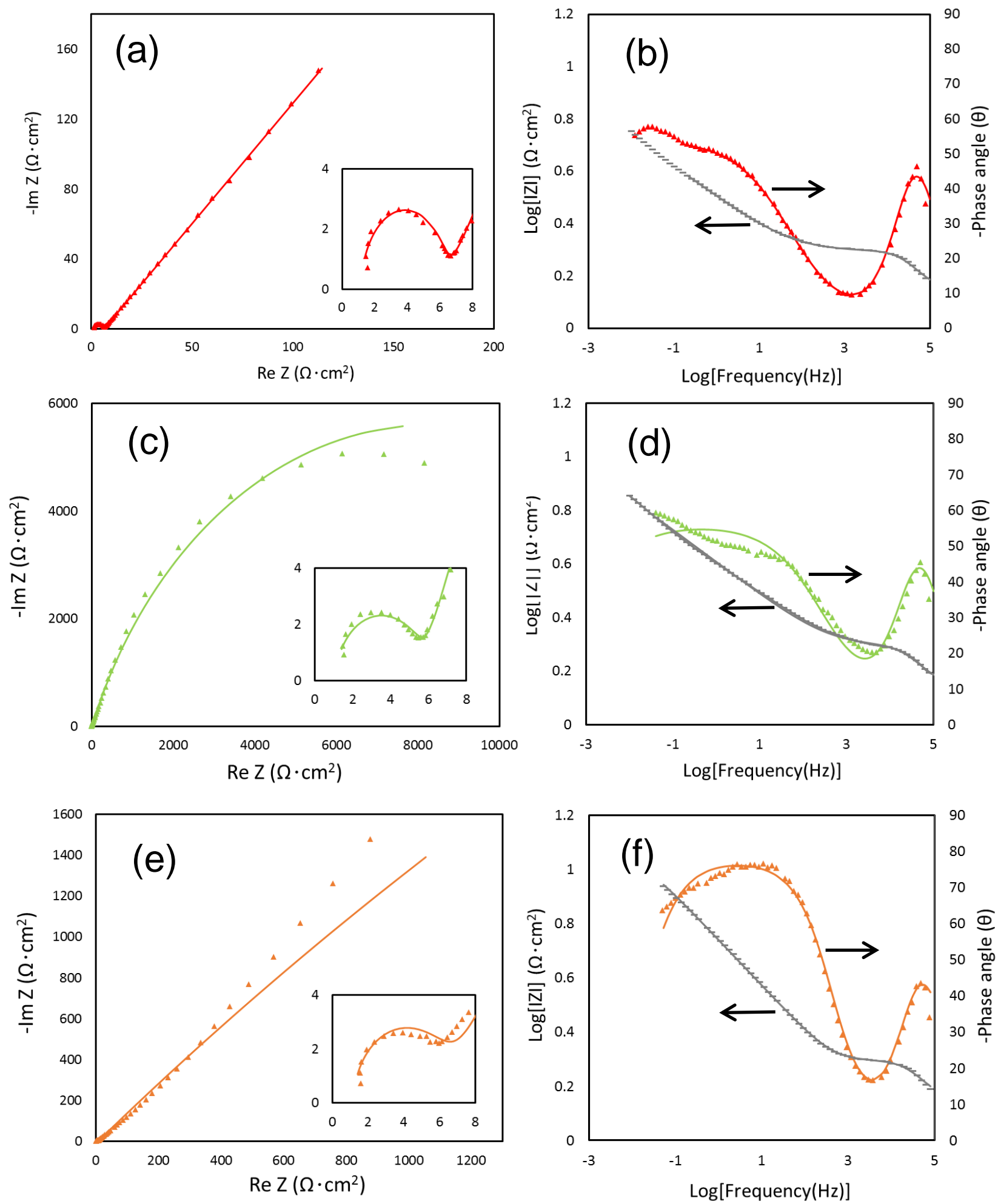

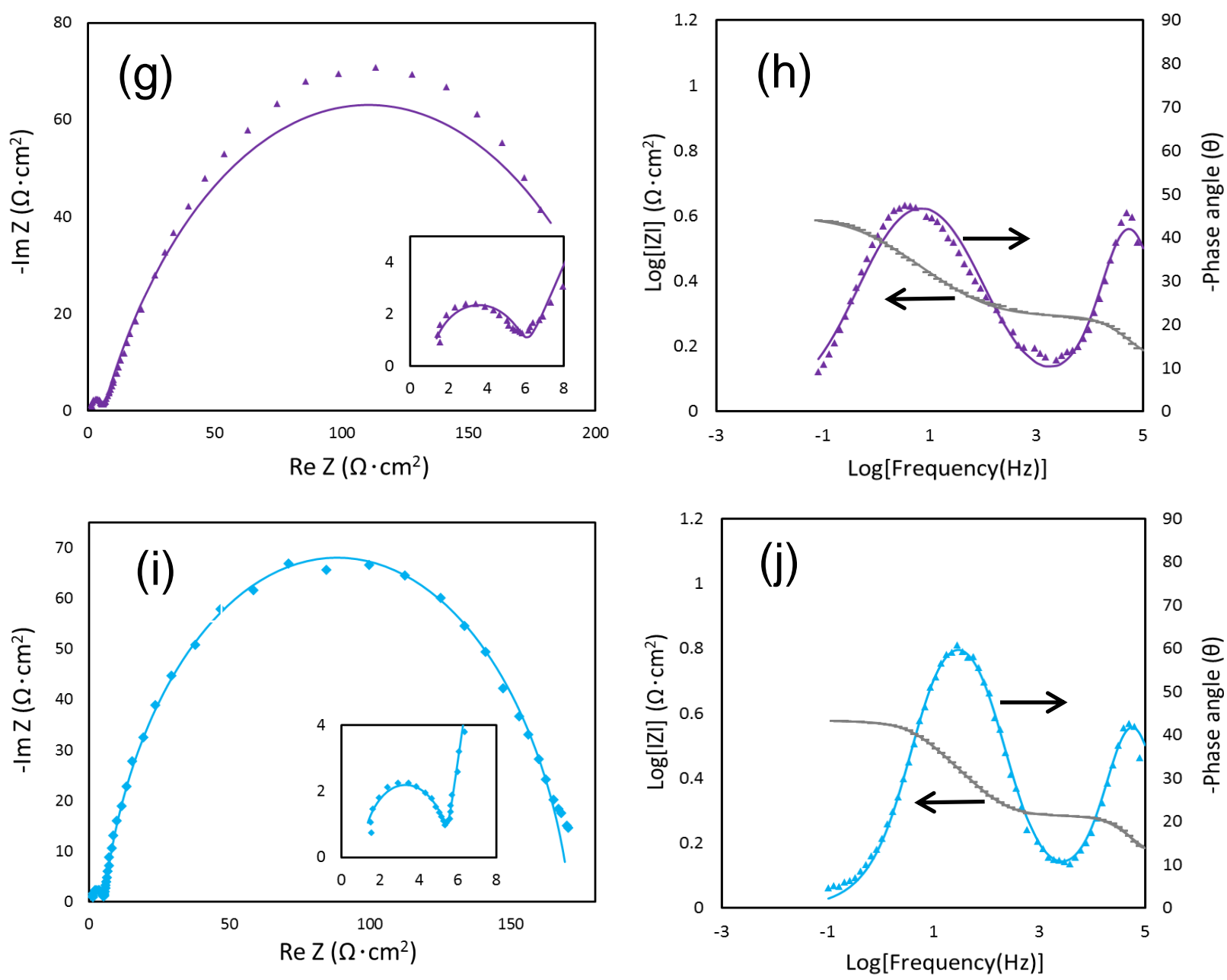

Figure S4. Nyquist and Bode plots of the electrochemical impedance spectra for various $\mathrm{MCo}_{2} \mathrm{O}_{4}$ $(\mathrm{M}=\mathrm{Mn}, \mathrm{Fe}, \mathrm{Co}, \mathrm{Ni}$, and $\mathrm{Zn})$ catalysts in $0.1 \mathrm{M} \mathrm{KOH}$ electrolyte at $1.6 \mathrm{~V}$ (vs. RHE). Experimental (markers) and simulated (full lines) Nyquist plots of (a) $\mathrm{Co}_{3} \mathrm{O}_{4}$, (c) $\mathrm{MnCo}_{2} \mathrm{O}_{4}$, (e) $\mathrm{FeCo}_{2} \mathrm{O}_{4}$, (g) $\mathrm{NiCo}_{2} \mathrm{O}_{4}$, and (i) $\mathrm{ZnCo}_{2} \mathrm{O}_{4}$ catalyst, and Bode plots of (b) $\mathrm{Co}_{3} \mathrm{O}_{4}$, (d) $\mathrm{MnCo}_{2} \mathrm{O}_{4}$, (f) $\mathrm{FeCo}_{2} \mathrm{O}_{4}$, (h) $\mathrm{NiCo}_{2} \mathrm{O}_{4}$, and (j) $\mathrm{ZnCo}_{2} \mathrm{O}_{4}$ catalyst. Range of investigated frequency is $100 \mathrm{kHz}-$ $0.1 \mathrm{~Hz}$. The high frequency region of the Nyquist plot is shown as an inset. 

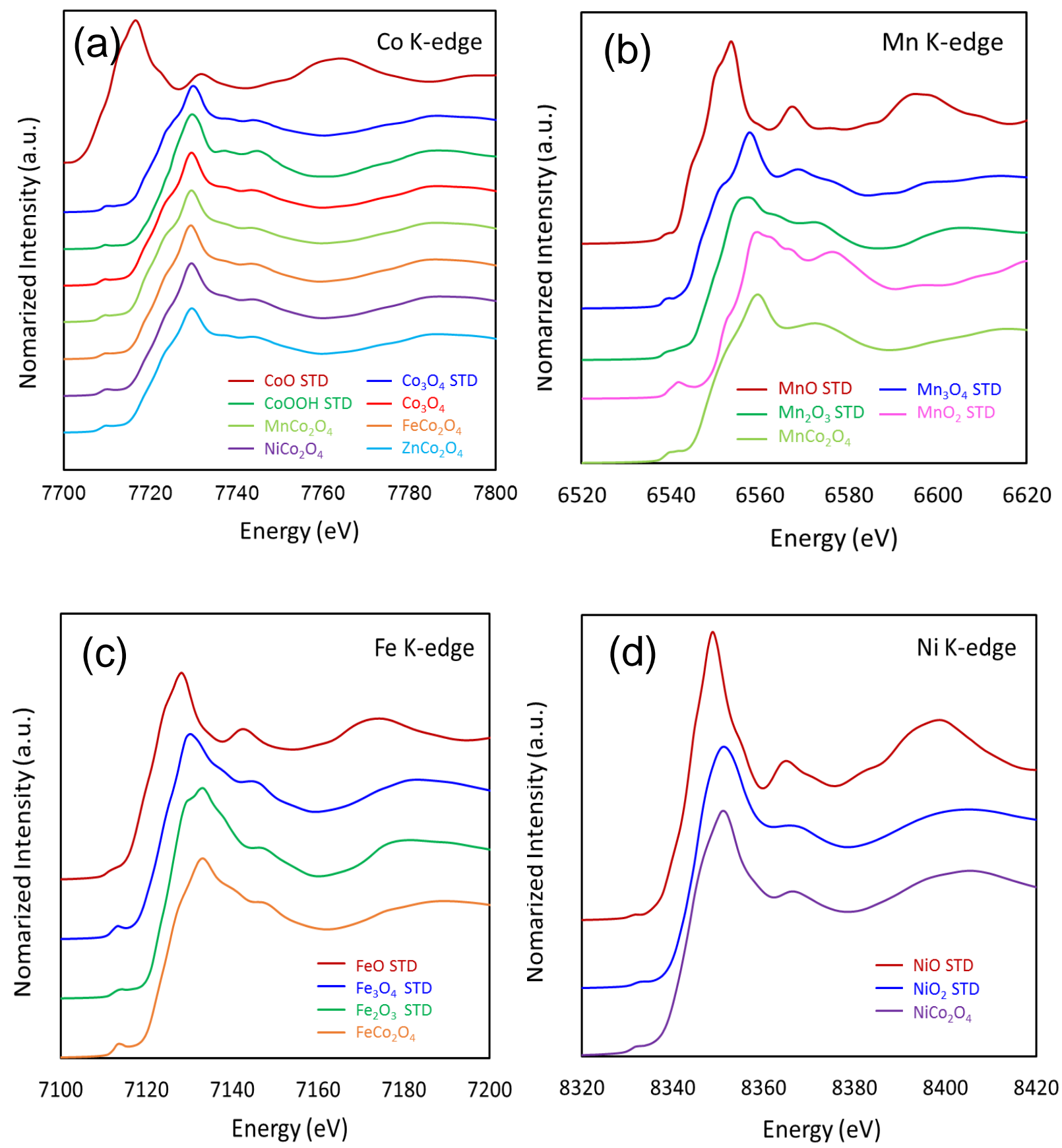


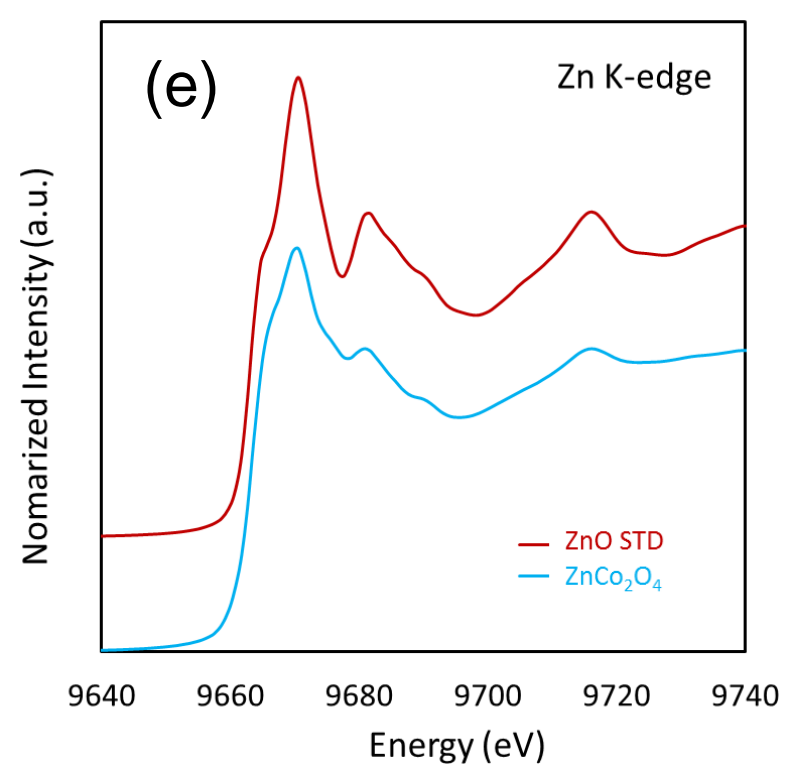

Figure S5. XANES spectra of (a) Co K-edge, (b) Mn K-edge, (c) Fe K-edge, (d) Ni $\mathrm{K}$-edge, and (e) $\mathrm{Zn} \mathrm{K}$-edge for various $\mathrm{MCo}_{2} \mathrm{O}_{4}(\mathrm{M}=\mathrm{Mn}, \mathrm{Fe}, \mathrm{Co}, \mathrm{Ni}$, and $\mathrm{Zn})$ catalysts and reference compounds. 


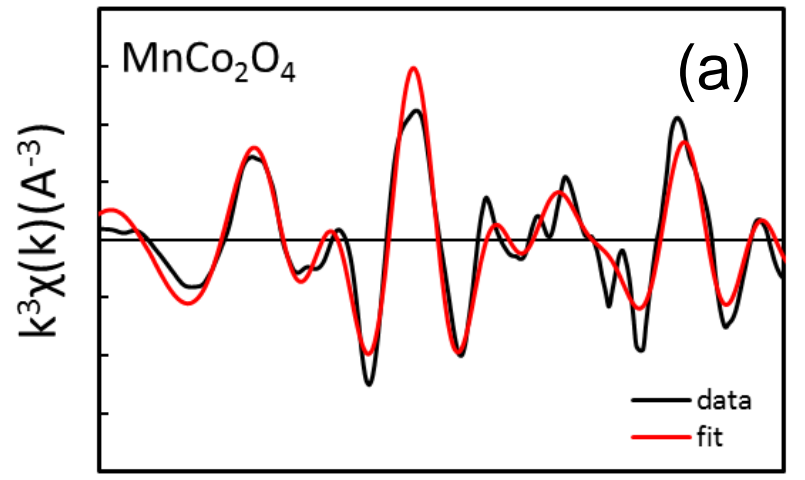

$\begin{array}{lllllllllll}2 & 3 & 4 & 5 & 6 & 7 & 8 & 9 & 10 & 11 & 12\end{array}$ Wavenumber $\left(\AA^{-1}\right)$
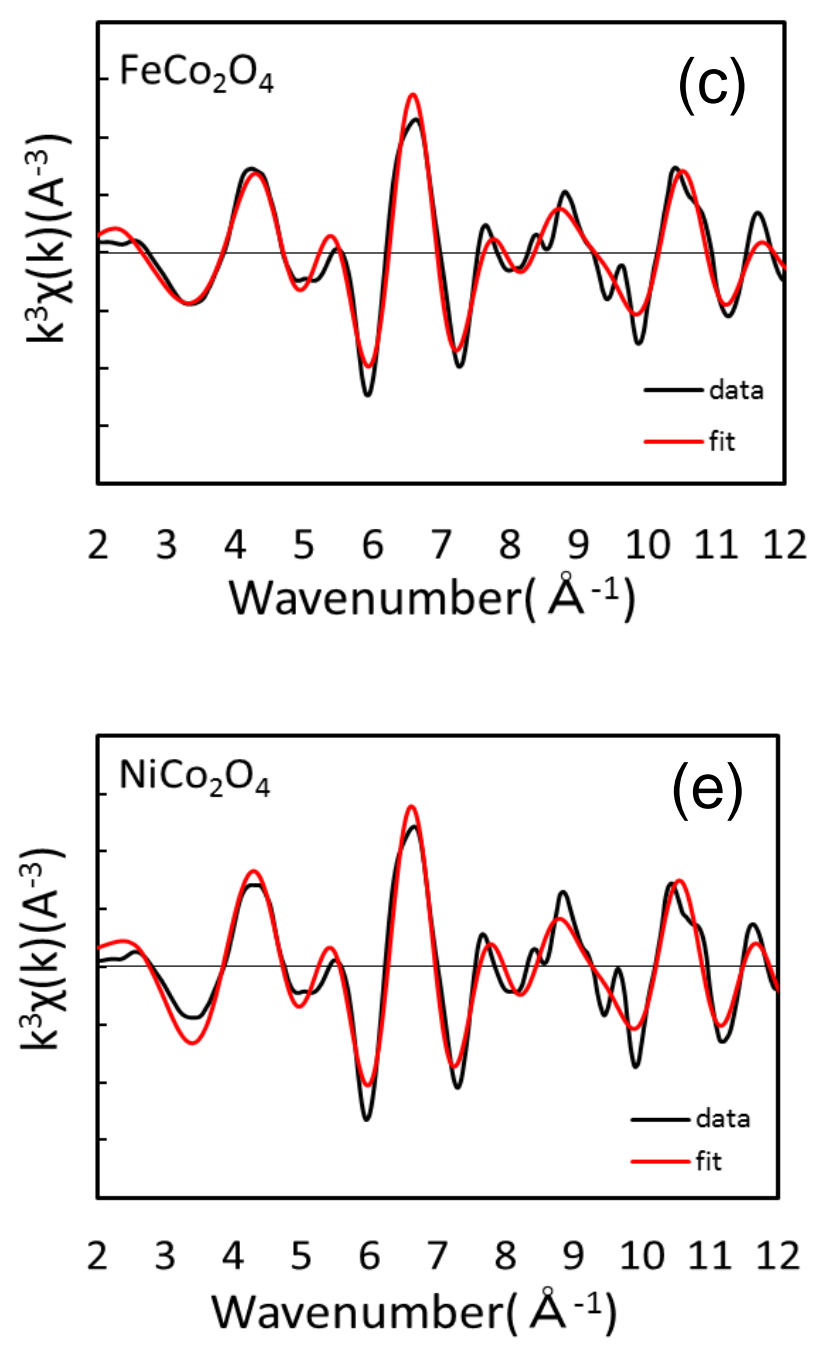
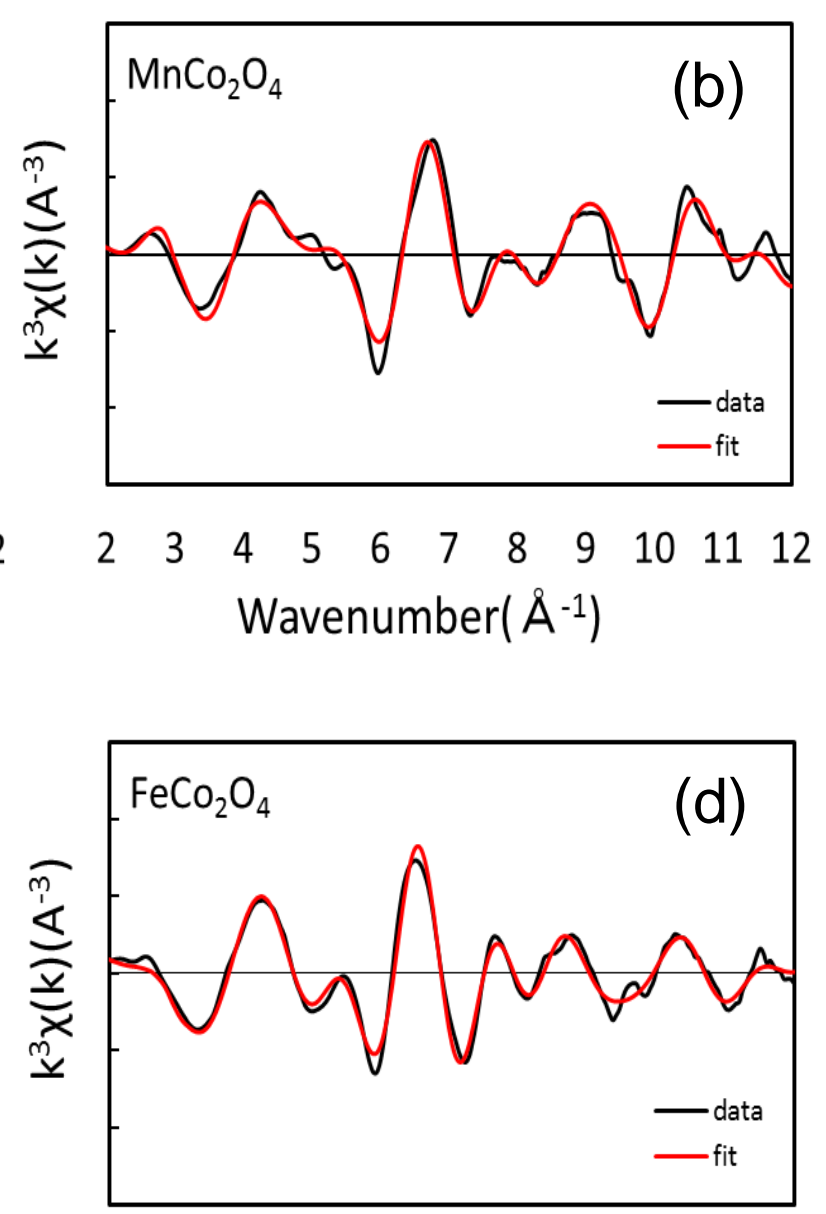

$\begin{array}{lllllllllll}2 & 3 & 4 & 5 & 6 & 7 & 8 & 9 & 10 & 11 & 12\end{array}$ Wavenumber $\left(\AA^{-1}\right)$

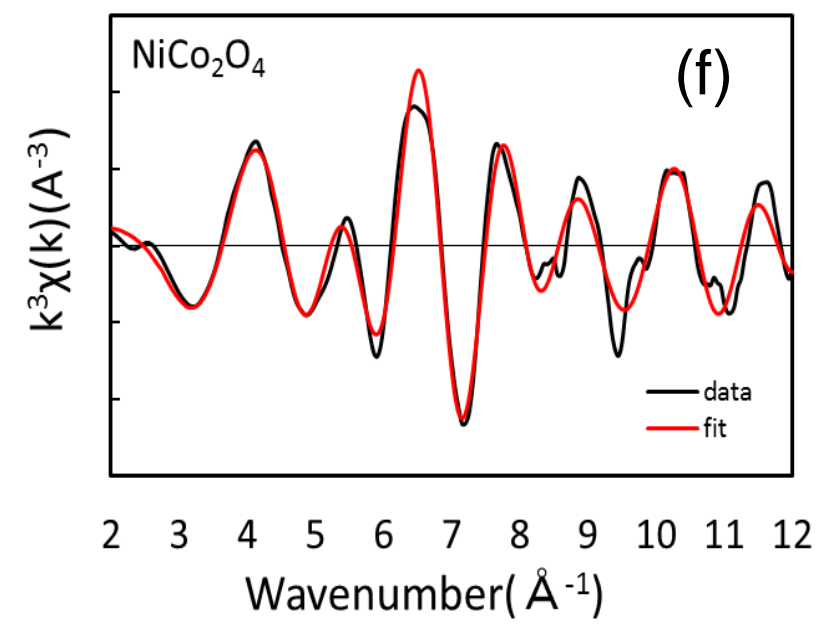




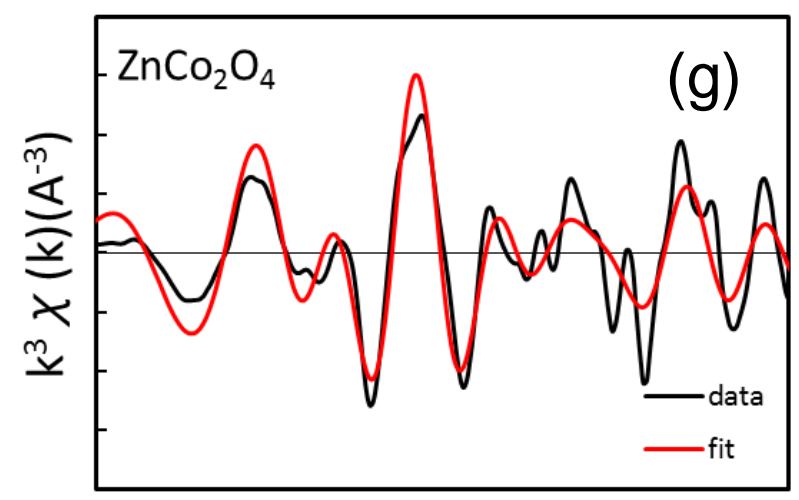

$\begin{array}{lllllllllll}2 & 3 & 4 & 5 & 6 & 7 & 8 & 9 & 10 & 11 & 12\end{array}$ Wavenumber $\left(\AA^{-1}\right)$

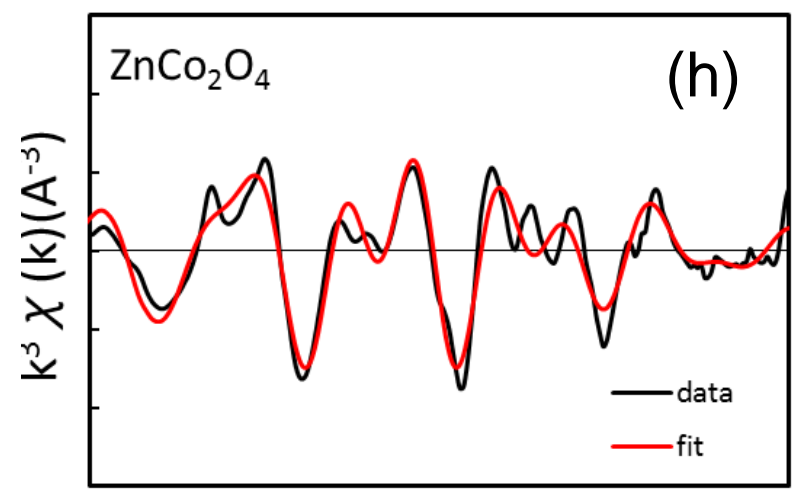

$\begin{array}{lllllllllll}2 & 3 & 4 & 5 & 6 & 7 & 8 & 9 & 10 & 11 & 12\end{array}$ Wavenumber $\left(\AA^{-1}\right)$

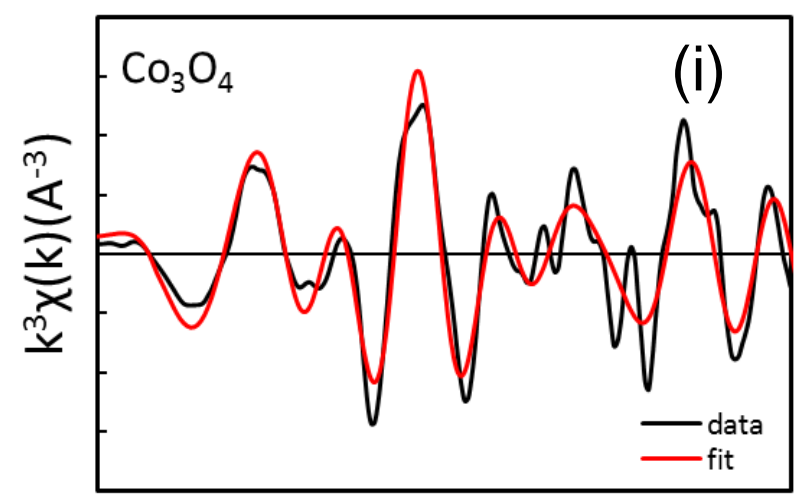

\section{$\begin{array}{lllllllllll}2 & 3 & 4 & 5 & 6 & 7 & 8 & 9 & 10 & 11 & 12\end{array}$ Wavenumber $\left(\AA^{-1}\right)$}

Figure S6. $k^{3} \chi(k)$ EXAFS spectra at the Co K-edge for (a) $\mathrm{MnCo}_{2} \mathrm{O}_{4}$, (c) $\mathrm{FeCo}_{2} \mathrm{O}_{4}$, (e) $\mathrm{NiCo}_{2} \mathrm{O}_{4}$, (g) $\mathrm{ZnCo}_{2} \mathrm{O}_{4}$, and (i) $\mathrm{Co}_{3} \mathrm{O}_{4}$ nanoparticles and at the (b) Mn K-edge, (d) $\mathrm{Fe}$ $\mathrm{K}$-edge, (f) $\mathrm{Ni} \mathrm{K}$-edge, and (h) $\mathrm{Zn} \mathrm{K}$-edge for the corresponding $\mathrm{MCo}_{2} \mathrm{O}_{4}$ nanoparticles coated by organic ligands (OAm, OA, and TOPO). The black solid lines represent the experimental data, and the best-fitted curves are shown as red solid lines. 

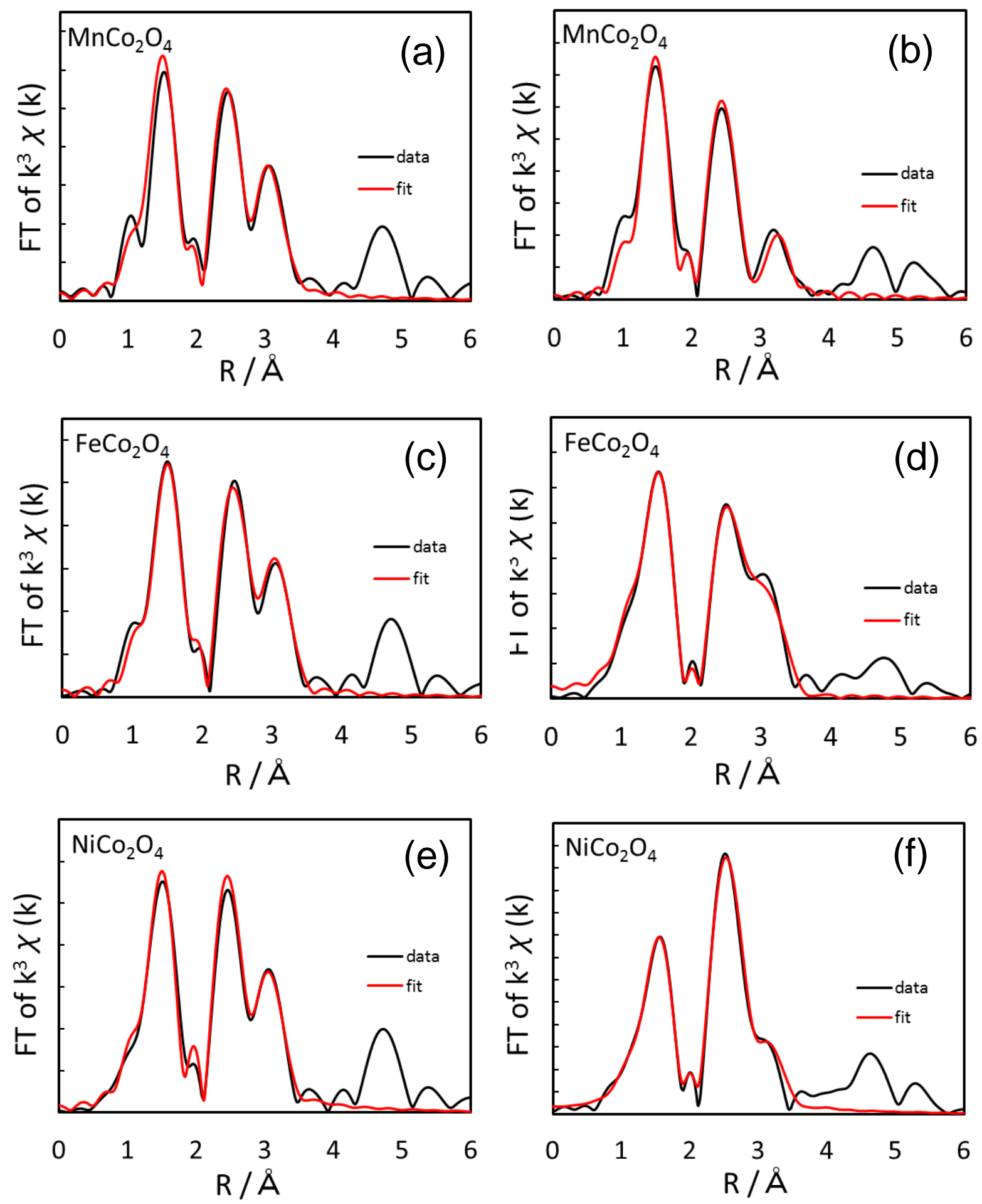

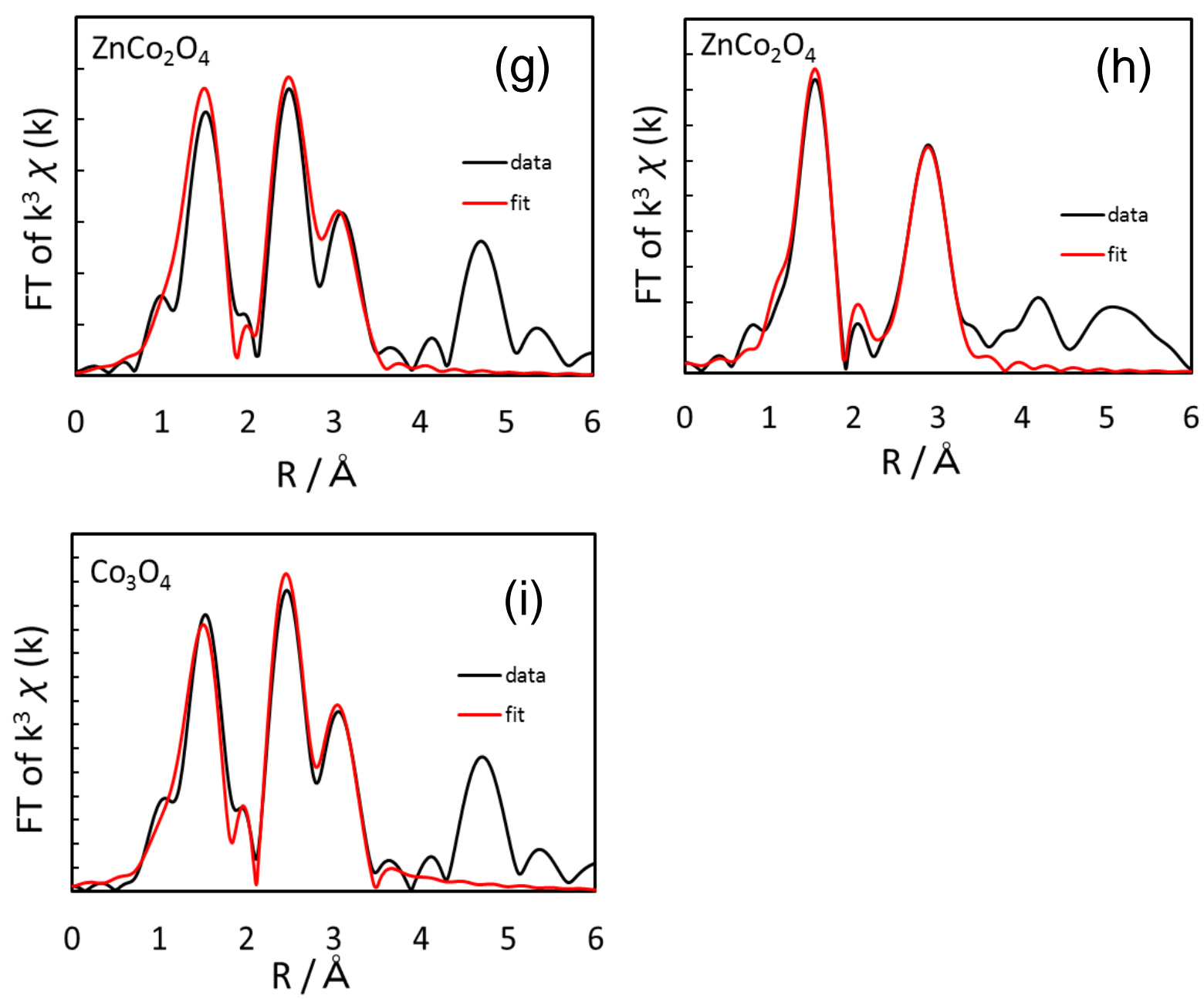

Figure S7. Fourier transforms at the Co K-edge for (a) $\mathrm{MnCo}_{2} \mathrm{O}_{4}$, (c) $\mathrm{FeCo}_{2} \mathrm{O}_{4}$, (e) $\mathrm{NiCo}_{2} \mathrm{O}_{4}$, (g) $\mathrm{ZnCo}_{2} \mathrm{O}_{4}$, and (i) $\mathrm{Co}_{3} \mathrm{O}_{4}$ nanoparticles and at the (b) Mn K-edge, (d) $\mathrm{Fe}$ $\mathrm{K}$-edge, (f) Ni K-edge, and (h) Zn K-edge for the corresponding nanoparticles coated by organic ligands. The black solid lines represent the experimental data, and the best-fitted curves are shown as red solid lines. 


\section{References}

(1) McCrory, C. C. L.; Jung, S.; Peters, J. C.; Jaramillo, T. F. Benchmarking Heterogeneous Electrocatalysts for the Oxygen Evolution Reaction. J. Am. Chem. Soc. 2013, 135, 16977-16987.

(2) Zhu, C.; Fu, S.; Du, D.; Lin, Y. Facilely Tuning Porous $\mathrm{NiCo}_{2} \mathrm{O}_{4}$ Nanosheets with Metal Valence-State Alteration and Abundant Oxygen Vacancies as Robust Electrocatalysts Towards Water Splitting. Chem. Eur. J. 2016, 22, 4000-4007.

(3) Liu, Y.; Ying, Y.; Fei, L.; Liu, Y.; Hu, Q.; Zhang, G.; Pang, S. Y.; Lu, W.; Mak, C. L.; Luo, X.; Zhou, L.; Wei, M.; Huang, H. Valence Engineering via Selective Atomic Substitution on Tetrahedral Sites in Spinel Oxide for Highly Enhanced Oxygen Evolution Catalysis. J. Am. Chem. Soc. 2019, 141, 8136-8145. 